\title{
Spin-exchange interactions of spin-one bosons in optical lattices: Singlet, nematic, and dimerized phases
}

\section{Citation}

Imambekov, Adilet, Mikhail Lukin, and Eugene Demler. 2003. "Spin-Exchange Interactions of Spin-One Bosons in Optical Lattices: Singlet, Nematic, and Dimerized Phases." Physical Review A 68 (6) (December 4). doi:10.1103/physreva.68.063602.

\section{Published Version}

doi:10.1103/physreva.68.063602

\section{Permanent link}

http://nrs.harvard.edu/urn-3:HUL.InstRepos:27945412

\section{Terms of Use}

This article was downloaded from Harvard University's DASH repository, and is made available under the terms and conditions applicable to Other Posted Material, as set forth at http:// nrs.harvard.edu/urn-3:HUL.InstRepos:dash.current.terms-of-use\#LAA

\section{Share Your Story}

The Harvard community has made this article openly available.

Please share how this access benefits you. Submit a story.

\section{Accessibility}




\title{
Spin-exchange interactions of spin-one bosons in optical lattices: Singlet, nematic, and dimerized phases
}

\author{
Adilet Imambekov, Mikhail Lukin, and Eugene Demler \\ Department of Physics, Harvard University, Cambridge, Massachusetts 02138, USA
}

(Received 8 June 2003; published 4 December 2003)

\begin{abstract}
We consider insulating phases of cold spin-1 bosonic particles with antiferromagnetic interactions, such as ${ }^{23} \mathrm{Na}$, in optical lattices. We show that spin-exchange interactions give rise to several distinct phases, which differ in their spin correlations. In two- and three-dimensional lattices, insulating phases with an odd number of particles per site are always nematic. For insulating states with an even number of particles per site, there is always a spin-singlet phase, and there may also be a first-order transition into the nematic phase. The nematic phase breaks spin rotational symmetry but preserves time reversal symmetry, and has gapless spin-wave excitations. The spin-singlet phase does not break spin symmetry and has a gap to all excitations. In onedimensional lattices, insulating phases with an odd number of particles per site always have a regime where translational symmetry is broken and the ground state is dimerized. We discuss signatures of various phases in Bragg scattering and time-of-flight measurements.
\end{abstract}

DOI: $10.1103 /$ PhysRevA.68.063602

PACS number(s): 03.75.Mn, 32.80.Pj, 71.35.Lk

\section{INTRODUCTION}

Modern studies of quantum magnetism in condensed matter physics go beyond explaining details of particular experiments on cuprate superconductors, heavy fermion materials, organic conductors, or related materials, and aim to develop general paradigms for understanding complex orders in strongly interacting many-body systems [1-10]. Spinor atoms in optical lattices provide a novel realization of quantum magnetic systems that have several advantages compared to their condensed matter counterparts, including precise knowledge of the underlying microscopic models, the possibility to control parameters of the effective lattice Hamiltonians, and the absence of disorder.

Degenerate alkali-metal atoms are generally considered as a weakly interacting gas due to the smallness of the scattering length compared to the interparticle separation [11]. The situation may change dramatically when either the atomic scattering length is changed by means of Feshbach resonance [12] or when an optical potential created by standing laser beams confines particles in the minima of the periodic potential and strongly enhances the effects of interactions. In the latter case, the existence of the nontrivial Mott insulating state of atoms in optical lattices, separated from the superfluid phase by the quantum phase transition (SI transition), was demonstrated recently in experiments [13-15]. Lowenergy (temperature) properties of spinless bosonic atoms in a periodic optical potential are well described by the BoseHubbard Hamiltonian [16]

$$
\mathcal{H}_{\mathrm{BH}}=-t \sum_{\langle i j\rangle}\left(a_{i}^{\dagger} a_{j}+a_{j}^{\dagger} a_{i}\right)-\mu \sum_{i} \hat{n}_{i}+\frac{U_{0}}{2} \sum_{i} \hat{n}_{i}\left(\hat{n}_{i}-1\right) \text {. }
$$

The parameters of Eq. (1) may be controlled by varying the intensity of laser beams, so one can go from the regime in which the kinetic energy dominates (weak periodic potential $t \gg U_{0}$ ) to the regime where the interaction energy is the most important part of the Hamiltonian (strong periodic po- tential $t \ll U_{0}$ ). For integer fillings (number of atoms per lattice site), the two regimes have superfluid and Mott insulating ground states, respectively, as can be obtained from the mean-field analysis of the Bose-Hubbard Hamiltonian $[16,17]$. In the superfluid phase, atoms are delocalized in the lattice, fluctuations in the number of atoms in each site are strong, and there is a phase coherence between different sites. In the insulating state, atoms are localized, fluctuations in the particle number at each site are suppressed, and there is a gap to all excitations. Such an insulating state represents a correlated many-body state of bosons, where strong interactions between atoms result in a new ground state of the system.

In conventional magnetic traps, the spins of atoms are frozen so effectively that they behave as spinless particles. In contrast, optically trapped atoms have extra spin degrees of freedom which can exhibit different types of magnetic orderings. In particular, alkali atoms have a nuclear spin $I=3 / 2$. The lower-energy hyperfine manifold has three magnetic sublevels and a total moment $S=1$. The various properties of such a condensate in a single trap were investigated [1823]. For example, for particles with antiferromagnetic interactions, such as ${ }^{23} \mathrm{Na}$, the exact ground state of an even number of particles in the absence of a magnetic field is a spin singlet described by a rather complicated correlated wave function [22]. However, when the number of particles in the trap is large, the energy gap separating the singlet ground state from the higher energy excited states is extremely small, and for the experiments of Ref. [24], the precession time of the classical mean-field ground state is of the order of the trap lifetime. So, experimental observation of the quantum spin phenomena in such systems is very difficult. To amplify quantum-spin effects one would like to have a system with a smaller number of particles and stronger interactions between atoms. Hence it is natural to consider an idea of $S=1$ atoms in an optical lattice, in which one can have a small number of atoms per lattice site (in the experiments of Ref. [14] this number was around 1-3) and relatively strong interactions between atoms. 


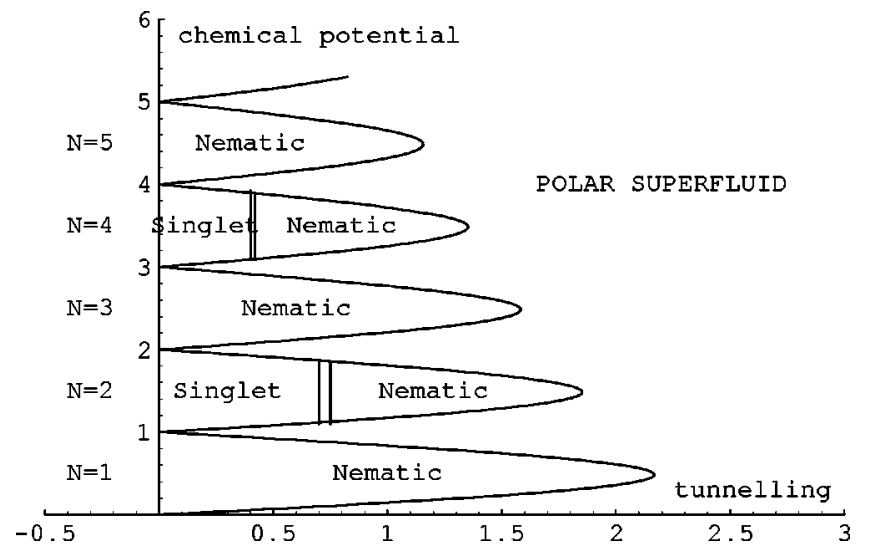

FIG. 1. General phase diagram for $S=1$ bosons in 2D and 3D optical lattice. Detailed discussion of the phase diagram, including explicit expressions for various phase boundaries, is given in Sec. VI.

In this paper we study bosonic $S=1$ atoms in optical lattices with spin symmetric confining potentials and antiferromagnetic interaction between atoms. We demonstrate that spin degrees of freedom result in a rich phase diagram by establishing the existence of several distinct insulating phases, which differ from each other by their spin correlations.

In the insulating state of bosons in an optical lattice fluctuations in the particle number on each site are suppressed but not frozen out completely. Virtual tunneling of atoms between neighboring lattice sites gives rise to effective spinexchange interactions that determine the spin structure of the insulating states (spin-exchange interactions for $S=1 / 2$ bosons in optical lattices were discussed previously in Refs. $[25,26])$.

We will show that in two- and three-dimensional lattices insulating states with an odd number of atoms per site are always nematic, whereas insulating states at even fillings are either singlet or spin nematic [27], depending on the parameters of the model. In one-dimensional systems even more exotic ground states should be realized, including the possibility of a spin-singlet dimerized phase that breaks lattice translational symmetry [28,29]. The two-dimensional (2D) and 3D general phase diagram, including singlet, nematic, and superfluid phases, is shown in Fig. 1. The extended version of this diagram, including a discussion of various transition lines, is presented in Sec. VI.

It is useful to point out that the lattice model for spin-1 bosons, which we analyze here, is very general and may also be applicable to systems other than cold atoms in optical lattices. For example, triplet superconductors in the strongcoupling limit may be described by a similar Hamiltonian, and some of the phases discussed in this article may correspond to non-BCS states of such superconductors [30].

The paper is organized as follows. In Sec. II we provide the derivation of the Hubbard-type Hamiltonian for spin-1 bosons in optical lattices starting from microscopic interactions between atoms, and describe some general properties of our model. In Sec. III we derive an effective spin Hamiltonian which is valid for any odd number of atoms per site $N$ in the limit of small tunneling between sites. We demonstrate the equivalence between our system and a Heisenberg model for $S=1$ spins on a lattice with biquadratic interactions and argue that the ground state is a nematic in two and three dimensions and is a dimerized singlet in 1D. In Sec. IV we derive an effective spin Hamiltonian for a system with $N$ $=2$ atoms per site, valid deep in the insulating regime, and use a mean-field approximation to determine the phase boundaries between isotropic and nematic phases. In Sec. V we derive the effective spin Hamiltonian for the limit of a large number of particles per site $N \gg 1$ and small tunneling, and discuss an isotropic-nematic transition for even $N$. In Sec. VI we summarize our results and review the global phase diagram for spin-1 bosons in optical lattices. Finally, in Sec. VII, we discuss approaches to an experimental detection of singlet and nematic insulating phases of $S=1$ bosons. Details of the technical calculations are presented in Appendixes $\mathrm{A}-\mathrm{D}$.

\section{DERIVATION OF BOSE-HUBBARD MODEL FOR SPIN-1 PARTICLES}

At low energies, scattering between two identical alkalimetal atoms with the hyperfine spins $S=1$ is well described by the contact potential [11]

$$
\begin{gathered}
\hat{V}\left(\mathbf{r}_{\mathbf{1}}-\mathbf{r}_{\mathbf{2}}\right)=\delta\left(\mathbf{r}_{\mathbf{1}}-\mathbf{r}_{\mathbf{2}}\right)\left(g_{0} \mathcal{P}_{0}+g_{2} \mathcal{P}_{2}\right), \\
g_{S}=4 \pi \hbar^{2} a_{S} / M .
\end{gathered}
$$

Here $\mathcal{P}_{S}$ is the projection operator for the pair of atoms into the state with total spin $S=0,2 ; a_{S}$ is the $s$-wave scattering length in the spin $S$ channel; and $M$ is the atomic mass. When writing Eq. (2) we used the fact that $s$-wave scattering of identical bosons in the channel with total spin 1 is not allowed by the symmetry of the wave function. Interaction (2) can be written using spin operators as

$$
V\left(\mathbf{r}_{\mathbf{1}}-\mathbf{r}_{\mathbf{2}}\right)=\delta\left(\mathbf{r}_{\mathbf{1}}-\mathbf{r}_{\mathbf{2}}\right)\left(\frac{g_{0}+2 g_{2}}{3}+\frac{g_{2}-g_{0}}{3} \mathbf{S}_{1} \cdot \mathbf{S}_{\mathbf{2}}\right)
$$

For example, in the case of ${ }^{23} \mathrm{Na}, g_{2}>g_{0}$, and we find effective antiferromagnetic interaction, as was originally discussed in Refs. [18,19].

Kinetic motion of ultracold atoms in the optical lattice is constrained to the lowest Bloch band when temperature and interactions are smaller than the band gap (this is the limit that we will consider from now on). Atoms residing on the same lattice site have identical orbital-wave functions and their spin-wave functions must be symmetric. If we introduce creation operators $a_{i \sigma}^{\dagger}$ for states in the lowest Bloch band localized on site $i$ and having spin components $\sigma=\{-1,0,1\}$, we can follow the approach of Ref. [16] and write the effective lattice Hamiltonian 


$$
\begin{aligned}
\mathcal{H}= & -t \sum_{\langle i j\rangle, \sigma}\left(a_{i \sigma}^{\dagger} a_{j \sigma}+a_{j \sigma}^{\dagger} a_{i \sigma}\right)+\frac{U_{0}}{2} \sum_{i} \hat{n}_{i}\left(\hat{n}_{i}-1\right) \\
& +\frac{U_{2}}{2} \sum_{i}\left(\vec{S}_{i}^{2}-2 \hat{n}_{i}\right)-\mu \sum_{i} \hat{n}_{i},
\end{aligned}
$$

where

$$
n_{i}=\sum_{\sigma} a_{i \sigma}^{\dagger} a_{i \sigma}
$$

is the total number of atoms on site $i$ and

$$
\vec{S}_{i}=\sum_{\sigma \sigma^{\prime}} a_{i \sigma}^{\dagger} \vec{T}_{\sigma \sigma^{\prime}} a_{i \sigma^{\prime}}
$$

is the total spin on site $i$ ( $\vec{T}_{\sigma \sigma^{\prime}}$ are the usual spin operators for spin-1 particles). The first term in Eq. (5) describes spinsymmetric tunneling between nearest-neighbor sites, the second term describes Hubbard repulsion between atoms, and the third term penalizes nonzero spin configurations on individual lattice sites. The origin of this spin-dependent term is the difference in scattering lengths for $S=0$ and $S=2$ channels as was discussed in Ref. [22]. Finally, the fourth term in Eq. (5) is the chemical potential that controls the number of particles in the system.

Hamiltonian (5) carries important constraints on possible spin states of the system. The first of them derives from the fact that the total spin of a system of $N$ spin-1 atoms cannot be bigger than $N$, so for each lattice site we have

$$
S_{i} \leqslant N_{i}
$$

The second constraint is imposed by the symmetry of the spin-wave function on each site

$$
S_{i}+N_{i}=(\text { even })
$$

Optical lattices produced by far detuned lasers with wavelength $\lambda_{i}=2 \pi /\left|\vec{k}_{i}\right|$ create an optical potential $V(\vec{r})$ $=\sum_{i} V_{i} \sin ^{2}\left(\vec{k}_{i} \cdot \vec{r}\right)$, with $\vec{k}_{i}$ being the wave vectors of laser beams. Using various orientations of beams, one can construct different geometries of the lattice. For the simple cubic lattice, the parameters of Eq. (5) can be estimated as

$$
\begin{aligned}
U_{2} & =\frac{2 \pi^{2}}{3} E_{R} \frac{a_{2}-a_{0}}{\lambda} x^{3 / 4}, \\
U_{0} & =\frac{2 \pi^{2}}{3} E_{R} \frac{a_{0}+2 a_{2}}{\lambda} x^{3 / 4}, \\
t & =\frac{2}{\sqrt{\pi}} E_{R} x^{3 / 4} e^{-2 x^{1 / 2}},
\end{aligned}
$$

where $E_{R}=\hbar^{2} k^{2} / 2 M$ is the recoil energy and $x=V_{0} / E_{R}$. Note that the ratio $U_{2} / U_{0}$ is fixed by the ratio of scattering lengths $a_{2} / a_{0}$ for all lattice geometries. Scattering lengths for ${ }^{23} \mathrm{Na}$ given in Ref. [31] are $a_{2}=(52 \pm 5) a_{B}$ and $a_{0}$

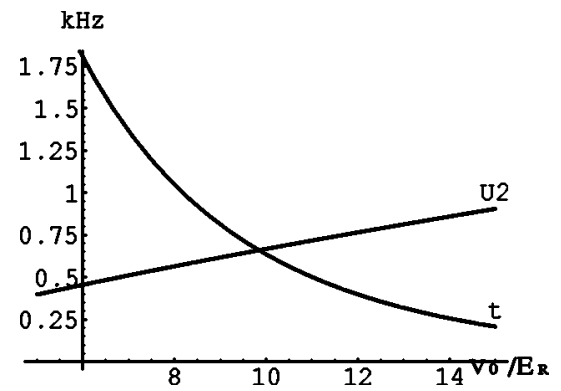

FIG. 2. $U_{2}$ and $t$ for ${ }^{23} \mathrm{Na}$ atoms in the simple cubic optical lattice created by three perpendicular standing laser beams with $\lambda$ $=985 \mathrm{~nm} . V_{0}$ is the strength of the optical potential and $E_{R}$ $=\hbar^{2} k^{2} / 2 M$ is the recoil energy. The ratio of the interaction terms in Eq. (5), $U_{2} / U_{0}$, is fixed by the ratio of the scattering lengths and is independent of the nature of the lattice $\left(U_{2} / U_{0} \approx 0.04\right.$ for $\left.{ }^{23} \mathrm{Na}\right)$.

$=(46 \pm 5) a_{B}$, where $a_{B}$ is the Bohr radius. This corresponds to $0<a_{2}-a_{0} \ll 2 a_{2}+a_{0}$, so the spin-dependent part of the interaction is much smaller than the spin-independent one. Throughout this paper we will always assume $0<U_{2} \ll U_{0}$. While applying the results of this paper for the case of ${ }^{23} \mathrm{Na}$, one should note that errors in the estimation of the exact value of $U_{2} / U_{0}$ are very big. While considering the spin structure of Mott insulating phases, we will assume that $U_{2} / U_{0}$ is small enough to see the interplay between tunneling and spin-dependent $U_{2}$ term before the superfluidinsulator transitions take place. The positions of superfluidinsulator transitions and the validity of this assumption will be discussed in detail in Sec. VI. We will use the value $U_{2} / U_{0}=0.04$ to make estimates of various phase boundaries. In Fig. 2 we show $U_{2} / \hbar$ and $t / \hbar$ as a function of the strength of the optical potential for a three-dimensional cubic lattice produced by red detuned lasers with $\lambda=985 \mathrm{~nm}$.

The superfluid-insulator transition is characterized by a change in fluctuations in particle number on individual lattice sites. When the spin-dependent interaction $\left(U_{2}\right)$ is much smaller than the usual Hubbard repulsion $\left(U_{0}\right)$, the superfluid-insulator transition is determined mostly by $U_{0}$. The spin-gap $U_{2}$ term, however, is important inside the insulating phase, where it competes with the spin-exchange interactions induced by small fluctuations in the particle number, and an interesting spin structure of the insulating states appears as a result of such competition. The spin structure of the insulating phases of spin-1 bosons in optical lattices will be explored in this paper.

In what follows we will often find it convenient to use particle creation operators that transform as vectors under spin rotations. Such a representation may be constructed as

$$
a_{z}^{\dagger}=a_{0}^{\dagger}, a_{x}^{\dagger}=\frac{\left(a_{-}\right)^{\dagger}-a_{+}^{\dagger}}{\sqrt{2}}, \quad a_{y}^{\dagger}=i \frac{\left(a_{-}\right)^{\dagger}+a_{+}^{\dagger}}{\sqrt{2}} .
$$

Operators $a_{\{x, y, z\}}$ satisfy the usual bosonic commutation relations, and they can be used to construct spin operators as

$$
S_{i a}=-i e_{a b c} a_{i b}^{\dagger} a_{i c}, \vec{S}_{i}^{2}=-\left(\delta_{b n} \delta_{\gamma m}-\delta_{b m} \delta_{\gamma n}\right) a_{b}^{\dagger} a_{\gamma} a_{n}^{\dagger} a_{m} .
$$


We can verify the transformation properties of $a_{\{x, y, z\}}$ by noting that

$$
\begin{aligned}
& {\left[S_{a}, a_{b}\right]=\left[-i e_{a p c} a_{p}^{\dagger} a_{c}, a_{b}\right]=i e_{a b c} a_{c},} \\
& {\left[S_{a}, a_{b}^{\dagger}\right]=\left[-i e_{a p c} a_{p}^{\dagger} a_{c}, a_{b}^{\dagger}\right]=i e_{a b c} a_{c}^{\dagger} .}
\end{aligned}
$$

Using these operators the hopping term in the Hamiltonian (5) may be rewritten as

$$
-t \sum_{\langle i j\rangle, p \in\{x, y, z\}}\left(a_{i p}^{\dagger} a_{j p}+a_{j p}^{\dagger} a_{i p}\right)
$$

and it is invariant under global spin rotations. We will use this property later to simplify calculations and classify eigenstates of effective interaction by the total spin of two neighboring sites.

\section{INSULATING STATE WITH AN ODD NUMBER OF ATOMS}

\section{A. Effective spin Hamiltonian for small $t$}

We start with the insulating state of the Hamiltonian (5) with an odd number $(N=2 n+1)$ of bosons per site in the limit $t=0$. The number of particles on each site is fixed, and the bosonic symmetry of the wave function requires that the spin in each site is odd. The interaction $U_{2}$ term is minimized when the spins take the smallest possible value $S$ $=1$. In this limit the energy of the system does not depend on the spin orientations on different sites. When $t$ is finite but small, we expect that we still have spin $S=1$ in each site, but that boson tunneling processes induce effective interactions between these spins. In this section we will compute such interactions in the lowest (second) order in $t$. We will also discuss conditions for which our effective Hamiltonian provides an adequate description of the system.

In the second-order perturbation theory in $t$, we generate only pairwise interactions between atoms on neighboring sites, so we can write the most general spin Hamiltonian for $S=1$ particles that preserves spin $\mathrm{SO}(3)$ symmetry

$$
\mathcal{H}=-J_{0}-J_{1} \sum_{\langle i j\rangle} \vec{S}_{i} \cdot \vec{S}_{j}-J_{2} \sum_{\langle i j\rangle}\left(\vec{S}_{i} \cdot \vec{S}_{j}\right)^{2} .
$$

Here $\langle i j\rangle$ labels near-neighbor sites on the lattice. Absence of the higher-order terms, such as $\left(\vec{S}_{i} \cdot \vec{S}_{j}\right)^{3}$, follows from the fact that the product of any three spin operators for an $S$ $=1$ particle can be expressed via the lower-order terms.

To find the exchange constants $J_{0,1,2}$, we need to consider virtual processes that create a state with $N_{i}=2 n, N_{j}=2 n$ +2 , and $N_{i}=2 n+2, N_{j}=2 n$. The difference in energy between the intermediate state and low-energy $S_{i}=S_{j}=1$ subspace is of order $U_{0}$, and while our subspace is much lower in energy, the second-order perturbation theory is valid.

It is convenient to rewrite the Hamiltonian (14) as

$$
\mathcal{H}=\epsilon_{0} \sum_{\langle i j\rangle} P_{i j}(0)+\epsilon_{1} \sum_{\langle i j\rangle} P_{i j}(1)+\epsilon_{2} \sum_{\langle i j\rangle} P_{i j}(2),
$$

$$
\begin{gathered}
\epsilon_{0}=-4 J_{2}+2 J_{1}-J_{0}, \\
\epsilon_{1}=-J_{2}+J_{1}-J_{0}, \\
\epsilon_{2}=-J_{2}-J_{1}-J_{0} .
\end{gathered}
$$

Here $P_{i j}(S)$ is a projection operator for a pair of spins on near-neighbor sites $i$ and $j$ into a state with total spin $S_{i}$ $+S_{j}=S(S=0,1,2)$. The equivalence of Eqs. (14) and (15) can be proven by noting simple operator identities for two spin-one particles

$$
\begin{gathered}
1=P_{i j}(0)+P_{i j}(1)+P_{i j}(2), \\
\left(\vec{S}_{i}+\vec{S}_{j}\right)^{2}=4+2 \vec{S}_{i} \cdot \vec{S}_{j}=2 P_{i j}(1)+6 P_{i j}(2), \\
\left(\vec{S}_{i}+\vec{S}_{j}\right)^{4}=16+16 \vec{S}_{i} \cdot \vec{S}_{j}+4\left(\vec{S}_{i} \cdot \vec{S}_{j}\right)^{2}=4 P_{i j}(1)+36 P_{i j}(2) .
\end{gathered}
$$

Note that states $\left|S_{i}=1, S_{j}=1 ; S_{i}+S_{j}=S\right\rangle$ have only the trivial degeneracy corresponding to possible projections of total spin $S$ on a fixed quantization axis $D_{S}=2 S+1$.

Since we know a general form of our effective Hamiltonian, we can compute $\epsilon_{0,1,2}$ by calculating the expectation values of energy for arbitrary states in the appropriate subspaces

$$
\begin{aligned}
\epsilon_{S}= & -t^{2} \sum_{m} \\
& \times \frac{\left|\left\langle m\left|\left(a_{i p}^{\dagger} a_{j p}+a_{j p}^{\dagger} a_{i p}\right)\right| S_{i}=1, S_{j}=1 ; S_{i}+S_{j}=S\right\rangle\right|^{2}}{E_{m}-E_{0}} .
\end{aligned}
$$

Here $E_{0}=2 U_{2}$ is the energy of the configuration with $N$ $=2 n+1$ bosons in each of the two wells and $E_{m}$ is the energy of the intermediate (virtual) states $m$ that have $2 n$ and $2 n+2$ bosons in the two wells, respectively. Both energies should be computed at zeroth order in $t$.

It is useful to note that the tunneling Hamiltonian is spin invariant; therefore, intermediate states in $m$ summation in Eq. (18) should also have total spin $S$. Another constraint on the possible states $m$ comes from the fact that the tunneling term can only change the spin on each site by \pm 1 since, in a Hilbert space of each well, operators $a_{i p}^{\dagger}, a_{i p}$ act as vectors, according to their transformational properties (12), (13).

Direct calculations in Appendix A give

$$
\begin{gathered}
\epsilon_{0}=-\frac{4 t^{2}(n+1)(2 n+3)}{3\left(U_{0}-2 U_{2}\right)}-\frac{16 t^{2} n(5+2 n)}{15\left(U_{0}+4 U_{2}\right)}, \\
\epsilon_{1}=-\frac{4 t^{2} n(5+2 n)}{5\left(U_{0}+4 U_{2}\right)}, \\
\epsilon_{2}=-\frac{28 t^{2} n(5+2 n)}{75\left(U_{0}+4 U_{2}\right)}-\frac{4\left(15+20 n+8 n^{2}\right)}{15\left(U_{0}+U_{2}\right)} .
\end{gathered}
$$

Combining Eqs. (15)-(21) we find 


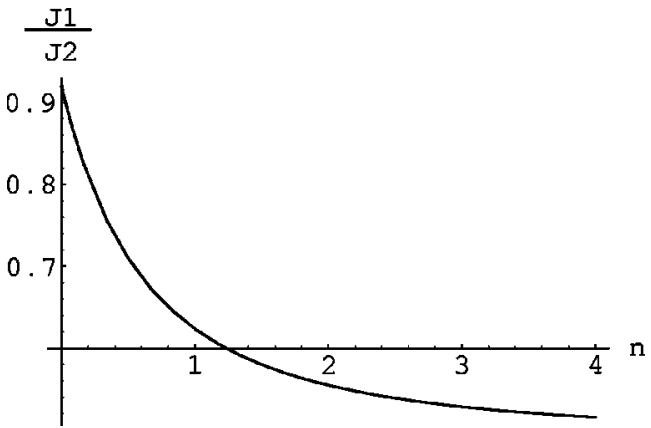

FIG. 3. Ratio $J_{1} / J_{2}$ for the effective spin Hamiltonian (14) for an odd number of bosons $N=2 n+1$ per site. $U_{2}=0.04 U_{0}$.

$$
\begin{aligned}
\frac{J_{0}}{t^{2}}= & \frac{4\left(15+20 n+8 n^{2}\right)}{45\left(U_{0}+U_{2}\right)}-\frac{4(1+n)(3+2 n)}{9\left(U_{0}+2 U_{2}\right)} \\
& +\frac{128(5+2 n)}{225\left(U_{0}+4 U_{2}\right)}, \\
\frac{J_{1}}{t^{2}}= & \frac{2\left(15+20 n+8 n^{2}\right)}{15\left(U_{0}+U_{2}\right)}-\frac{16(5+2 n) n}{75\left(U_{0}+4 U_{2}\right)}, \\
\frac{J_{2}}{t^{2}}= & \frac{2\left(15+20 n+8 n^{2}\right)}{45\left(U_{0}+U_{2}\right)}+\frac{4(1+n)(3+2 n)}{9\left(U_{0}-2 U_{2}\right)} \\
& +\frac{4 n(5+2 n)}{225\left(U_{0}+4 U_{2}\right)} .
\end{aligned}
$$

It will turn out that the ratio between $J_{1}$ and $J_{2}$ determines the magnetic ground state and its dependence on $n$ is quite fast, as shown in Fig. 3 .

We now discuss limitations of the Hamiltonian (14) with Eq. (22). In the insulating state with exactly one boson per site, near-neighbor interactions always have the form (14). Explicit expressions for the $J$ 's given in Eq. (22) only apply in the limit $t \ll U_{0}$. When $t$ becomes comparable to $U_{0}$ (but we are still in the insulating phase), higher-order terms become important, including the possibility of spin coupling beyond the near-neighbor sites. In the insulating state with more than one boson per site $(N=2 n+1, n>0)$, we have an additional constraint: we should be able to neglect configurations with spins on individual sites higher than 1. Matrix elements for scattering into such states are of the order of $(N t)^{2} / U_{0}$ [see Eq. (22)], and their energy is set by $U_{2}$. Therefore, the Hamiltonian (14) applies only when $N t$ $\ll\left(U_{0} U_{2}\right)^{1 / 2}$, which is well within the insulating state when $U_{2} \ll U_{0}$ (the SI transition takes place for $N t \sim U_{0}$ ).

\section{B. Phase diagram}

To understand the nature of the Hamiltonian (14) in the relevant regime of parameters $J_{2}>J_{1}>0$, it is useful to start by considering a two-site problem

$$
\mathcal{H}_{12}=-J_{1} \vec{S}_{1} \cdot \vec{S}_{2}-J_{2}\left(\vec{S}_{1} \cdot \vec{S}_{2}\right)^{2}
$$

TABLE I. Eigenstates of the two-site problem (23).

\begin{tabular}{cccc}
\hline \hline$S_{\text {tot }}$ & $\vec{S}_{1} \cdot \vec{S}_{2}$ & $\left(\vec{S}_{1} \cdot \vec{S}_{2}\right)^{2}$ & Energy \\
\hline 0 & -2 & 4 & $2 J_{1}-4 J_{2}$ \\
1 & -1 & 1 & $J_{1}-J_{2}$ \\
2 & 1 & 1 & $-J_{1}-J_{2}$ \\
\hline \hline
\end{tabular}

with $S_{1}=S_{2}=1$. Eigenstates of Eq. (23) can be classified according to the value of the total spin $S_{\text {tot }}$, and their energies may be computed using $2 \vec{S}_{1} \cdot \vec{S}_{2}=S_{\text {tot }}\left(S_{\text {tot }}+1\right)-4$. Two spin-one particles can combine into $S_{\text {tot }}=0,1$, and 2. The $J_{1}$ term in Eq. (23) favors maximizing $\vec{S}_{1} \cdot \vec{S}_{2}$ by making the fully polarized $S_{\text {tot }}=2$ state. By contrast, the $J_{2}$ term favors maximizing $\left(\vec{S}_{1} \cdot \vec{S}_{2}\right)^{2}$ by forming a singlet state $S_{\text {tot }}=0$ (see Table I). So, the latter term acts as an effective antiferromagnetic interaction for this spin one system, and it dominates for $J_{2}>J_{1}$. If we go beyond a two-site problem and consider a large lattice, we see that each pair of near-neighbor sites wants to establish a singlet configuration when $J_{2}>J_{1}$. However, because one cannot form singlets on two different bonds that share the same site, some interesting spin order, whose precise nature will depend on the lattice and dimensionality, will appear.

\section{Phase diagram for $d=1$}

From the discussion above we see the conflict intrinsic to the Hamiltonian (14): each bond wants to have a singlet-spin configuration, but singlet states on the neighboring bonds are not allowed. There are two simple ways to resolve this conflict.

(A) Construct a state that mixes $S=0$ and $S=2$ on each bond but can be repeated on neighboring bonds.

(B) Break translational symmetry and favor singlets on every second bond.

At the mean-field level, a solution of the type $\mathrm{A}$ is given by

$$
|N\rangle=\prod_{i}\left|S_{i}=1, m_{i}=0\right\rangle
$$

This can be established by noting that for any neighboring pair of sites we indeed have a superposition of $S=0$ and $S$ $=2$ states

$$
\begin{aligned}
\mid S_{i} & \left.=1, m_{i}=0\right\rangle\left|S_{j}=1, m_{j}=0\right\rangle \\
& =-\frac{1}{\sqrt{3}}\left|S_{\mathrm{tot}}=0\right\rangle+\sqrt{\frac{2}{3}}\left|S_{\mathrm{tot}}=2, m_{\mathrm{tot}}=0\right\rangle .
\end{aligned}
$$

State (24) describes a nematic state that has no expectation value of any component of the $\operatorname{spin}\left\langle S_{i}^{x, y, z}=0\right\rangle$, but spin symmetry is broken since $\left\langle\left(S_{i}^{x}\right)^{2}\right\rangle=\left\langle\left(S_{i}^{y}\right)^{2}\right\rangle=1 / 2$ and $\left\langle\left(S_{i}^{z}\right)^{2}\right\rangle=0$. It is useful to point out the similarity between wave function (25) that mixes singlet and quintet states on each bond, and a classical antiferromagnetic state for spin$1 / 2$ particles that mixes spin singlets and triplets on each 
bond. Coleman's theorem [32] (the quantum analog of Mermin-Wagner theorem) forbids the breaking of spin symmetry in $d=1$, even at $T=0$. However, a spin-singlet gapless ground state that has a close connection to the nematic state (24) has been proposed in Refs. [33,34] for $J_{2}$ close to $J_{1}$.

The simplest way to construct a solution of type B is to take

$$
|D\rangle=\prod_{i=2 n}\left|S_{i}=1, S_{i+1}=1, S_{i}+S_{i+1}=0\right\rangle .
$$

Such a dimerized solution has exact spin singlets for pairs of sites $2 n$ and $2 n+1$, but pairs of sites $2 n$ and $2 n-1$ are in a superposition of $S=0,1$, and 2 states.

According to the variational wave functions (24) and (26), the dimerized solution becomes favorable over a nematic one only for $J_{2} / J_{1}>3 / 2$ in $d=1$. However, numerical simulations [35] showed that for $J_{2}>J_{1}$, the ground state is always dimerized. It is a spin singlet and has a gap to all spin excitations. This means that the variational wave function (26) may only be taken as a caricature of the true ground state, although it captures key aspects of it, such as broken translational symmetry and the absence of spin-symmetry breaking.

\section{Phase diagram for $d=2,3$}

The nematic state for the Hamiltonian (14) in a simple cubic lattice $(d=3)$ for $J_{2}>J_{1}$ has been discussed using mean-field calculations [36], a semiclassical approach [37], and numerically [38]. Finally, the recent work of Tanaka et al. [39] provided a rigorous proof of the existence of the nematic order at least in some part of this region, which satisfies $2.66 J_{1}>J_{2} \geqslant 2 J_{1}$. The variational state for the nematic order may again be given by Eq. (24) and its mean-field energy is $E_{N}^{\mathrm{MF}}=-2 J_{2}$. It is important to emphasize, however, that the actual ground state is sufficiently different from its mean-field version (24). It is possible to write down dimerized states with energy expectation lower than $-2 J_{2}$; however, numerical results [38] suggest that the ground state does not break translational symmetry. The way to obtain a more precise ground-state wave function is to include quantum fluctuations near the mean-field state, as was done in Ref. [37]. Hence, the mean-field wave function (24) does not provide a good approximation of the ground-state energy of the nematic state. Nevertheless, it is useful for the discussion of order parameter and broken symmetries of the nematic state.

In the nematic state, spin-space rotational group $\mathrm{O}(3)$ is broken, though time-reversal symmetry is preserved. The order parameter for the nematic state is a tensor

$$
Q_{a b}=\left\langle S_{a} S_{b}\right\rangle-\frac{\delta_{a b}}{3}\left\langle S^{2}\right\rangle .
$$

In the absence of ferromagnetic order $\left\langle S_{a} S_{b}\right\rangle=\left\langle S_{b} S_{a}\right\rangle$; hence, $Q_{a b}$ is a traceless symmetric matrix. The minimum energy of Eq. (14) is achieved for $Q_{a b}$ that has two identical eigenvalues, which corresponds to a uniaxial nematic [40].
Then, the tensor $Q_{a b}$ can be written using a unit vector $\vec{d}$ as

$$
Q_{a b}=Q\left(d_{a} d_{b}-\frac{1}{3} \delta_{a b}\right)
$$

Vector $\vec{d}$ is defined up to the direction (i.e., $\pm \vec{d}$ are equivalent) and corresponds to the director order parameter [40]. For the mean-field state (24), the director $\vec{d}$ can also be defined from the condition that locally our system is an eigenstate of the operator $\vec{d} \cdot \vec{S}$ with eigenvalue zero. However, such a definition may not be applied generally.

The nematic phase behaves in many aspects as antiferromagnetic [41], the direction of $\vec{d}$ being analogous to staggered magnetization. Namely, in weak magnetic fields, $\vec{d}$ aligns itself in the plane perpendicular to magnetic field, and spin-wave excitations have linear dispersion [34], with velocity

$$
c=\sqrt{2 z J_{2}\left(J_{2}-J_{1}\right)}
$$

Nematic phases for the system of spin-1 particles have been considered before in literature [33-39,41] so we will not discuss them here more extensively.

\section{INSULATING STATES WITH TWO ATOMS PER SITE}

In this section we consider an insulating state of two bosons per site. Possible spin values for individual sites are $S=0$ and $S=2$. In the limit $t=0$, the interaction part of the Hamiltonian (the $U_{2}$ term) is minimized when $S=0$. The amplitude for creating $S=2$ states, as well as the exchange energy of the latter, is of the order of $t^{2} / U_{0}$. So, when $t$ is of the order of $\left(U_{0} U_{2}\right)^{1 / 2}$ or larger, we may no longer assume that we only have singlets in individual sites, and we need to include $S=2$ configurations in our discussion. This regime is still inside the insulating phase for small enough $U_{2} / U_{0}$ (the superfluid-insulator transition takes place for $z t \sim U_{0}$ ). In this section we will assume that $U_{2} / U_{0}$ is small enough, so that $S=2$ becomes important in the insulating phase, before the transition to superfluid. More careful consideration of the superfluid transition line and comparison with the case of ${ }^{23} \mathrm{Na}$ will be presented in Sec. VI. In Sec. IV A we exactly solve the problem for two wells. In Sec. IV B we derive an effective Hamiltonian that takes into account competition between spin gaps of individual sites, that favors $S=0$ everywhere, and exchange interactions between neighboring sites that favor proliferation of $S=2$ states. A mean-field solution of the effective magnetic Hamiltonian is considered in Sec. IV $\mathrm{C}$ and we find first-order quantum phase transition from isotropic to nematic phase. We discuss collective excitations in Secs. IV D and IV E and the effects of magnetic field in Sec. IV F. We note that the state with $N=2$ has an advantage over states with higher $N$ from an experimental point of view since it has no three-body decays. 


\section{A. Two site problem: Exact solution}

To construct an effective magnetic Hamiltonian for this system, we note that in the second order in $t$ it can be written as a sum of interaction terms for all near-neighbor sites (identical for all pairs of sites). These pairwise interactions can be found by solving a two-well problem and finding the appropriate eigenvalues and eigenvectors in the second order in $t$.

The Hilbert space for two sites with two atoms in each well is given by the direct sum of the following subspaces:

$$
\begin{aligned}
& \left|E_{1}\right\rangle=\left|N_{1}=2, N_{2}=2, S_{1}=S_{2}=0, S_{1}+S_{2}=0\right\rangle, \\
& \left|E_{2}\right\rangle=\left|N_{1}=2, N_{2}=2, S_{1}=S_{2}=2, S_{1}+S_{2}=0\right\rangle, \\
& \left|E_{3}\right\rangle=\left|N_{1}=2, N_{2}=2, S_{1}=0, S_{2}=2, S_{1}+S_{2}=2\right\rangle, \\
& \left|E_{4}\right\rangle=\left|N_{1}=2, N_{2}=2, S_{1}=2, S_{2}=0, S_{1}+S_{2}=2\right\rangle, \\
& \left|E_{5}\right\rangle=\left|N_{1}=2, N_{2}=2, S_{1}=S_{2}=2, S_{1}+S_{2}=2\right\rangle, \\
& \left|E_{6}\right\rangle=\left|N_{1}=2, N_{2}=2, S_{1}=S_{2}=2, S_{1}+S_{2}=1\right\rangle, \\
& \left|E_{7}\right\rangle=\left|N_{1}=2, N_{2}=2, S_{1}=S_{2}=2, S_{1}+S_{2}=3\right\rangle, \\
& \left|E_{8}\right\rangle=\left|N_{1}=2, N_{2}=2, S_{1}=S_{2}=2, S_{1}+S_{2}=4\right\rangle .
\end{aligned}
$$

The hopping term in Eq. (5) conserves total spin; therefore, the energy in each subspace does not depend on the $z$ component of $S_{1}+S_{2}$, and states $\left|E_{6}\right\rangle,\left|E_{7}\right\rangle$, and $\left|E_{8}\right\rangle$ form orthogonal subspaces that do not mix with any other states. We can then use formula analogous to Eq. (18) to calculate corrections to the energies of these states in the second order in $t$ (see Appendix B for details):

$$
\begin{aligned}
& \tilde{\epsilon}_{6}=6 U_{2}-4 \frac{t^{2}}{U_{0}}, \\
& \tilde{\epsilon}_{7}=6 U_{2}-4 \frac{t^{2}}{U_{0}}, \\
& \tilde{\epsilon}_{8}=6 U_{2}-12 \frac{t^{2}}{U_{0}} .
\end{aligned}
$$

In Eq. (30) we used $U_{2} \ll U_{0}$ and neglected $U_{2}$ relative to $U_{0}$ in denominators of the exchange terms.

Boson tunneling can connect two subspaces $\left|E_{1}\right\rangle$ and $\left|E_{2}\right\rangle$ and three subspaces $\left|E_{3}\right\rangle,\left|E_{4}\right\rangle$, and $\left|E_{5}\right\rangle$ (only states with the same component of $S^{z}$ have tunneling matrix elements). Thus, the energies and eigenstates should be found by diagonalizing the matrix

$$
\mathcal{H}_{\beta \alpha}=E_{0 \alpha} \delta_{\alpha \beta}-t^{2} \sum_{m} \frac{\left\langle\beta\left|\left(a_{1 p}^{\dagger} a_{2 p}+a_{2 p}^{\dagger} a_{1 p}\right)\right| m\right\rangle\left\langle m\left|\left(a_{1 p}^{\dagger} a_{2 p}+a_{2 p}^{\dagger} a_{1 p}\right)\right| \alpha\right\rangle}{E_{m}-E_{0 \alpha}} .
$$

Here $E_{0 \alpha}$ is the energy of the state $\alpha$ in the zeroth order in $t$ : $E_{01}=0, E_{02}=6 U_{2}, E_{03}=3 U_{2}, E_{04}=3 U_{2}$, and $E_{05}=6 U_{2}$. Summation over $m$ covers intermediate states that have one and three bosons in the two wells, have the same total spin as states $\alpha$ and $\beta$, and have spins in individual wells that differ from $\alpha$ and $\beta$ by \pm 1 . The explicit calculation presented in Appendix B gives the matrix $\mathcal{H}_{\beta \alpha}$ and its eigenvalues. Energies of the states with total spin zero are

$$
\begin{aligned}
& \tilde{\epsilon}_{1}=3 U_{2}-\frac{6 t^{2}}{U_{0}}-\sqrt{\left(3 U_{2}-\frac{6 t^{2}}{U_{0}}\right)^{2}+40 \frac{U_{2} t^{2}}{U_{0}}} \\
& \tilde{\epsilon}_{2}=3 U_{2}-\frac{6 t^{2}}{U_{0}}+\sqrt{\left(3 U_{2}-\frac{6 t^{2}}{U_{0}}\right)^{2}+40 \frac{U_{2} t^{2}}{U_{0}}}
\end{aligned}
$$

Energies of the states with $S=2$ are

$$
\begin{gathered}
\tilde{\epsilon}_{3}=3 U_{2}-4 \frac{t^{2}}{U_{0}}, \\
\tilde{\epsilon}_{4}=\frac{1}{2}\left(9 U_{2}-12 t^{2}+\sqrt{144 \frac{t^{4}}{U_{0}^{2}}+40 \frac{t^{2}}{U_{0}} U_{2}+9 U_{2}^{2}}\right),
\end{gathered}
$$

$$
\tilde{\epsilon}_{5}=\frac{1}{2}\left(9 U_{2}-12 t^{2}-\sqrt{144 \frac{t^{4}}{U_{0}^{2}}+40 \frac{t^{2}}{U_{0}} U_{2}+9 U_{2}^{2}}\right),
$$

and each of these states is fivefold degenerate. Energies $\tilde{\epsilon}_{1}-\tilde{\epsilon}_{8}$ are shown in Fig. 4.

For $U_{2}>0$ the lowest-energy state is a total spin singlet that has some mixture of $S=2$ states in individual wells

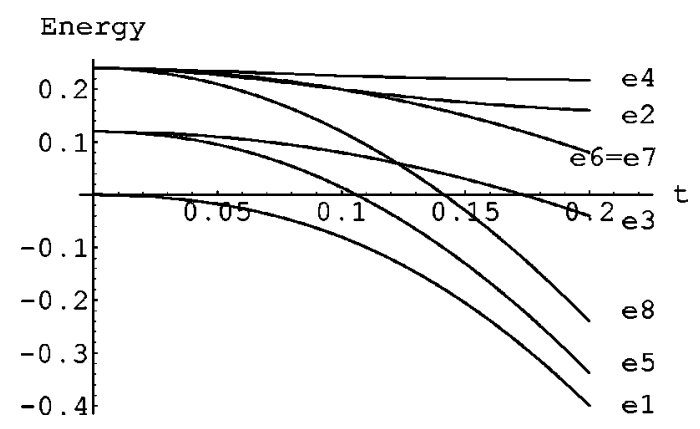

FIG. 4. Eigenstates of the effective spin Hamiltonian for a two site problem with two atoms per site. Energy and $t$ are measured in units of $U_{0}$, and we assumed $U_{2}=0.04 U_{0}$. The lowest energy states correspond to total spin $S=0(e 1), S=2(e 5)$, and $S=4(e 8)$. 
when $t$ is nonzero. The next favorable state has total spin 2, $\left|E_{5}\right\rangle$. When the value of $t$ is increasing, the ferromagnetic state $\left|E_{8}\right\rangle$ becomes the third low lying state. At this point, we have solved the problem for two wells, taking into account competition between hopping and the $U_{2}$ term (overall Hilbert space for two wells is 36 dimensional).

\section{B. Effective spin Hamiltonian for an optical lattice}

In the previous subsection we used perturbation theory in tunneling $t$ to study the problem of two sites with two atoms in each well. If we label the two sites 1 and 2 , in the second order in $t$ the effective Hamiltonian can be written as

$$
\begin{aligned}
\mathcal{H}_{12}= & 3 U_{2}\left[P\left(S_{1}=2\right)+P\left(S_{2}=2\right)\right] \\
& +|\alpha\rangle_{1}|\beta\rangle_{2} J_{\alpha, \beta ; \gamma, \delta}\left\langle\gamma | _ { 1 } \left\langle\left.\delta\right|_{2} .\right.\right.
\end{aligned}
$$

Here $P\left(S_{\{1,2\}}=2\right)$ are projection operators into states with spin $S=2$ on sites 1 and 2 and $J_{\alpha, \beta ; \gamma, \delta}$ gives exchange interactions that arise from virtual tunneling processes into states with particle numbers $\left(n_{1}=1, n_{2}=3\right)$ and $\left(n_{1}=3, n_{2}\right.$ $=1)$. The second term of Eq. (34) includes all initial states $\left(|\gamma\rangle_{1}\right.$ and $|\delta\rangle_{2}$ for sites 1 and 2, respectively) and all final states $\left(|\alpha\rangle_{1}\right.$ and $\left.|\beta\rangle_{2}\right)$.

Generalization of the effective spin Hamiltonian (34) for the case of optical lattice is obviously

$$
\mathcal{H}=3 U_{2} \sum_{i} P\left(S_{i}=2\right)+\sum_{\langle i j\rangle}|\alpha\rangle_{i}|\beta\rangle_{j} J_{\alpha, \beta ; \gamma, \delta}\left\langle\gamma | _ { i } \left\langle\left.\delta\right|_{j} .\right.\right.
$$

This Hamiltonian is linear in $U_{2}$ and, therefore, can be written as a sum of the bond terms

$$
\begin{gathered}
\mathcal{H}=\sum_{\langle i j\rangle} \widetilde{\mathcal{H}}_{i j}, \\
\widetilde{\mathcal{H}}_{i j}=\frac{3 U_{2}}{z}\left[P\left(S_{i}=2\right)+P\left(S_{j}=2\right)\right] \\
+|\alpha\rangle_{i}|\beta\rangle_{j} J_{\alpha, \beta ; \gamma, \delta}\left\langle\gamma | _ { i } \left\langle\left.\delta\right|_{j} .\right.\right.
\end{gathered}
$$

Individual terms $\widetilde{\mathcal{H}}_{i j}$ differ from Eq. (34) only by rescaling $U_{2} \rightarrow U_{2} / z$, where $z$ is the coordination number of the lattice. We did not give explicit expressions for $J_{\alpha, \beta ; \gamma, \delta}$ in the basis of eigenstates of individual spins $S_{i}$ and $S_{j}$ but in the basis of eigenstates of the total spin of the pair (29), expressions for $J_{\alpha, \beta ; \gamma, \delta}$ can be obtained from eigenstates and eigenvalues of Eq. (34) [see Eqs. (30)-(33) and Appendix B] (with a rescaled $U_{2}$ ). Therefore, we can write

$$
\widetilde{\mathcal{H}}_{i j}=\sum_{\alpha, \beta, S_{z}^{\alpha}}\left|E_{\alpha}^{i j}, S_{z}^{\alpha}\right\rangle \widetilde{\mathcal{H}}_{\alpha \beta}\left\langle E_{\beta}^{i j}, S_{z}^{\alpha}\right|,
$$

where states $\left|E_{\alpha}^{i j}, S_{z}^{\alpha}\right\rangle$ have been defined in Eqs. (29). Expressing $\left|E_{\alpha}^{i j}, S_{z}\right\rangle \quad$ via states $\mid N_{i}=2, S_{i}=\{0,2\}, S_{i z}$ $\left.=-S_{i} \cdots S_{i}\right\rangle$ using known Clebsch-Gordon coefficients
$\left|E_{\alpha}^{i j}, S_{z}\right\rangle=\sum_{S_{i z}^{\alpha} S_{j z}^{\alpha}} C_{S_{i}^{\alpha}, S_{i z}^{\alpha} ; S_{j}^{\alpha}, S_{j z}^{\alpha}}^{S^{\alpha}, N_{i}^{\alpha}}\left|N_{i}=2, S_{i}^{\alpha}, S_{i z}^{\alpha}\right\rangle\left|N_{j}=2, S_{j}^{\alpha}, S_{j z}^{\alpha}\right\rangle$,

we can write the Hamiltonian (35) as

$$
\hat{H}=\sum_{\langle i j\rangle}|\alpha\rangle_{i}|\gamma\rangle_{j} \mathcal{H}_{\alpha, \beta ; \gamma, \delta}\left\langle\beta | _ { i } \left\langle\left.\delta\right|_{j},\right.\right.
$$

where states $|\alpha\rangle-|\delta\rangle$ belong to the set $\{S=0\},\left\{S=2, S_{z}\right.$ $=-2, \ldots, 2\}$, and $\mathcal{H}_{\alpha, \beta ; \gamma, \delta}$ is given by proper rotation of $\tilde{\mathcal{H}}_{\alpha, \beta}$.

\section{Phase diagram from the mean-field calculation}

In this section we study the phase diagram of the system described by the Hamiltonian (38) using translational invariant variational wave functions. Such a mean-field approach gives correct ferromagnetic and antiferromagnetic states for Heisenberg Hamiltonians in $d \geqslant 2$, so we expect it to be applicable in our case. We think that this approach successfully captures the main features of the system: first-order transition between the spin gapped and the nematic phases, the nature of the order parameter in the nematic phase, and elementary excitations in both phases. However, we cannot rule out the possibility of more exotic phases that fall outside of our variational wave functions, for example, the dimerized phase discussed in Ref. [28], and numerical calculations are required to study if such phases will actually be present.

As we saw in the previous chapter, energy of the two-well problem is minimized when total spin is 0 . However, energy on all bonds cannot be minimized simultaneously, so we cannot solve a problem exactly for a lattice. We use a mean-field approach to overcome this difficulty, taking variational wave function

$$
\begin{aligned}
|\Psi\rangle= & \prod_{i}\left(c_{0,0}\left|N_{i}=2, S=0, S_{z}=0\right\rangle\right. \\
& \left.+\sum_{m=-2, \ldots, 2} c_{2, m}\left|N_{i}=2, S=2, S_{z}=m\right\rangle\right), \\
& \left|c_{0,0}\right|^{2}+\sum_{m=-2, \ldots, 2}\left|c_{2, m}\right|^{2}=1 .
\end{aligned}
$$

Now we can evaluate expectation value of energy over variational state (39) and find the ground state numerically.

We parametrize Eq. (40) as

$$
\begin{gathered}
c_{0,0}=\cos \theta, c_{2, m}=\sin \theta a_{m}, \\
\sum_{m=-2, \ldots, 2}\left|a_{m}\right|^{2}=1 .
\end{gathered}
$$

In Appendix $\mathrm{C}$ we demonstrate that for a region of $\theta$ where the mean-field energy is minimized, $\left[a_{m}\right]$ has the form, up to $\mathrm{SU}(2)$ rotations [42],

$$
\left[a_{m}\right]=(0,0,1,0,0)^{T} .
$$




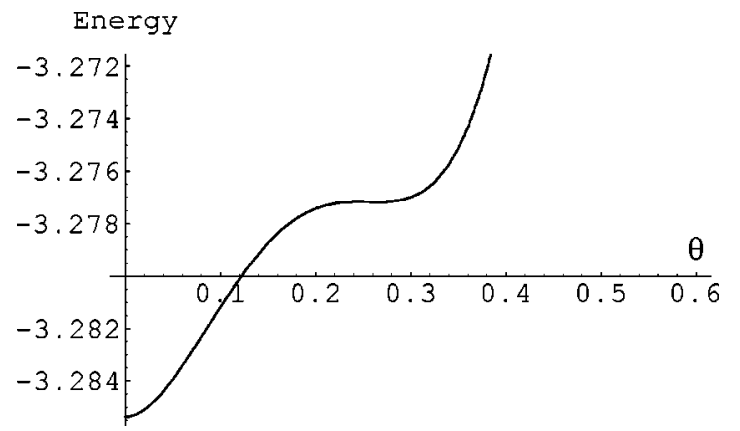

FIG. 5. Dependence of the energy functional (43) on $\theta$ when $z t^{2} /\left(U_{0} U_{2}\right) \approx 0.4928$ (energy is given per lattice site in units of $\left.U_{2}\right)$.

Mean-field energy does not depend on $\vec{d}$ and we find in the region of interest the energy per lattice site to be

$$
\begin{aligned}
E[\theta]= & 3 U_{2} \sin ^{2} \theta+\frac{z t^{2}}{12 U_{0}}(-51+4 \cos 2 \theta+7 \cos 4 \theta \\
& -8 \sqrt{2} \sin 2 \theta+4 \sqrt{2} \sin 4 \theta) .
\end{aligned}
$$

One can immediately see that if we try to expand this expression near $\theta=0$, there is no linear term, but second- and third-order terms are present, which indicates that by changing the parameters of the Hamiltonian, we will have a firstorder quantum phase transition, at which the value of $\theta$ that minimizes the energy changes discontinuously. This is typical for ordinary nematics [40] since in Landau expansion third order terms are not forbidden by $\vec{d} \rightarrow-\vec{d}$ symmetry. The reason why our transition is first order can be traced back to the fact that mean-field energy has terms which mix $c_{0,0}$ and $c_{2, m}$ in odd powers, i.e., $c_{0,0} c_{2,-2}\left(c_{2,-1}^{*}\right)^{2}$, and overall $\mathrm{U}(1)$ symmetry does not prohibit odd powers of $\theta$ in Eq. (43).

Since the phase transition is first order, it is characterized by several regimes. First, when $t$ is small, the global energy minimum is at $t=0$ and there are no other local minima, i.e., we have spin singlets in all individual sites. Then, when condition $z t_{-}^{2} /\left(U_{0} U_{2}\right) \approx 0.4928$ is satisfied, a local minimum appears at $\theta_{-} \approx 0.25$, see Fig. 5. As we continue increasing $t$, the minimum at nonzero $\theta$ becomes deeper, and eventually at $z t_{c}^{2} /\left(U_{0} U_{2}\right)=1 / 2$ the global minimum of Eq. (43) is reached

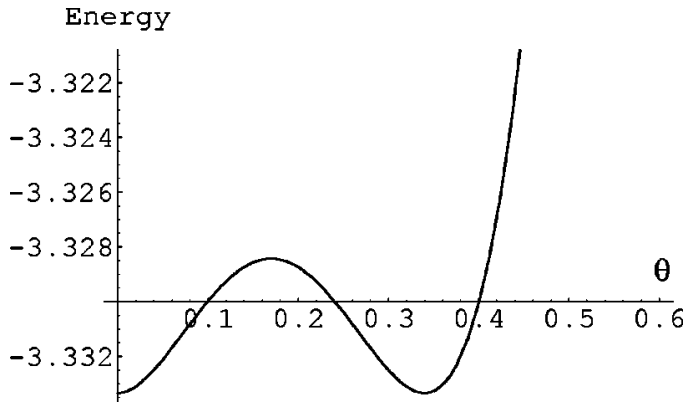

FIG. 6. Dependence of the energy functional (43) on $\theta$ when $z t^{2} /\left(U_{0} U_{2}\right)=1 / 2$ (energy is given per lattice site per lattice site in units of $U_{2}$ ).

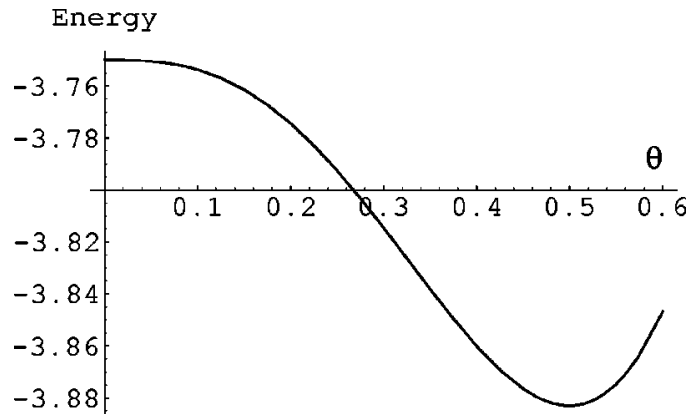

FIG. 7. Dependence of the energy functional (43) on $\theta$ when $z t^{2} /\left(U_{0} U_{2}\right)=9 / 16=0.5625$ (energy is given per lattice site in units of $U_{2}$ ).

for $\sin \theta_{c}=1 / 3$ (see Fig. 6). However, there is still a local minimum at $\theta=0$. If we keep increasing $t$, the minimum at $\theta=0$ becomes completely unstable at $z t_{+}^{2} /\left(U_{0} U_{2}\right)=9 / 16$ and there is only one minimum at $\theta_{+} \approx 0.5$ (see Fig. 7 ). As we increase $t$ further, $\sin \theta_{+}$continues to grow, approaching the value $\sin \theta_{\infty}=(2 / 3)^{1 / 2}$ (see Fig. 8). It is useful to point out that when $t$ is changed in experiments (e.g., by changing the strength of the optical potential [43]), we expect that the system will not switch between the singlet and nematic phases at $t_{c}$, but will remain in the appropriate metastable local minimum until it becomes completely unstable. So, in experiments with increasing $t$, the transition from the singlet to the nematic states will occur at $t_{+}$, and in experiments with decreasing $t$, the transition from the nematic to the singlet state will take place at $t_{-}$. We note, however, that the difference between different $t$ is quite small.

In Fig. 9 we show the phase diagram for the insulating phase with $N=2$, including a true first-order transition line at $t_{c}$ and limits of metastability at $t_{-}$and $t_{+}$. The superfluidinsulator transition line is shown as a guide for the eye for the case of small enough $U_{2} / U_{0}$; its exact position will be presented in Sec. VI. It is useful to point out that in the discussion above we used a canonical ensemble (fixed number of particles) rather than a grand canonical ensemble (fixed chemical potential) to discuss the singlet to nematic phase transition. However, intermediate states that contribute

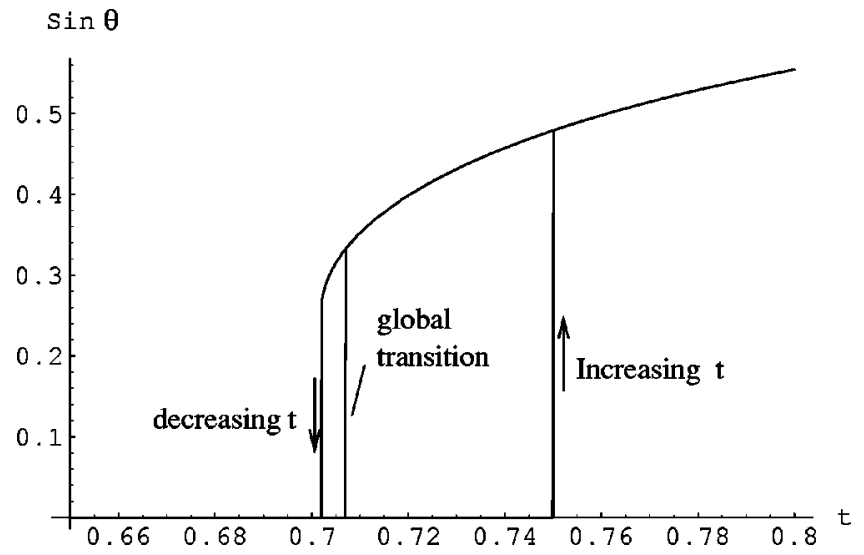

FIG. 8. Dependence of $\sin \theta$ in Eq. (43) on $t$, measured in units of $\sqrt{U_{0} U_{2} / z}$. 


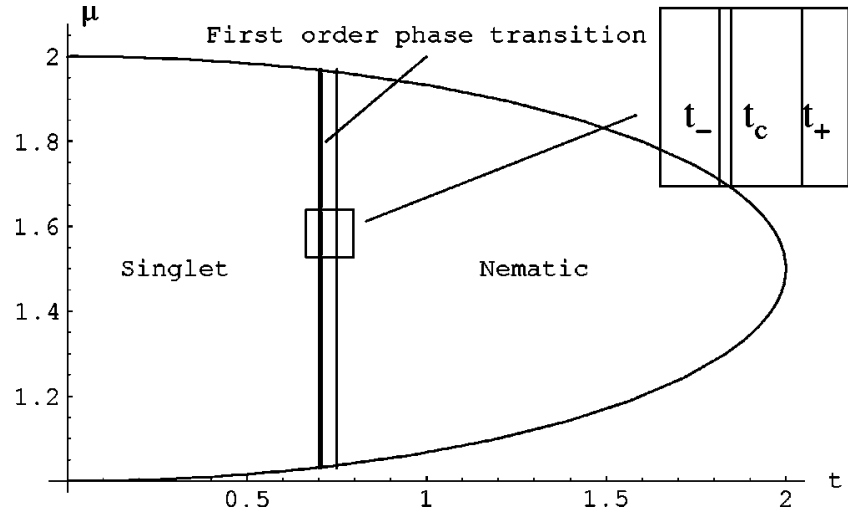

FIG. 9. Mott phase diagram for $N=2 . \mu$ is measured in units of $U_{0}, t$ is measured in units of $\sqrt{U_{0} U_{2} / z} . t_{c}$ marks the actual firstorder phase transition and $t_{-}$and $t_{+}$correspond to limits of metastability. The superfluid-insulator transition line is presented as a guide to the eye and will be discussed in Sec. VI.

to exchange interactions always involve one particle and one hole. Hence, their energy does not depend on the chemical potential. This explains why the singlet to nematic phase boundary in Fig. 9 does not depend on $\mu$. It is consistent with our physical intuition that insulating states have a certain number of particles, but their chemical potential $\mu$ is not well defined as long as $\mu$ is inside the Mott gap. In the discussion presented in this section, we assumed that the system remains deep in the insulating phase and the superfluid to insulator transition does not preempt the isotropic to nematic transition inside the insulating lobe. Precise conditions under which this is justified will be given in Sec. VI.

\section{Quantum fluctuations corrections for the spin singlet state}

For small enough $t$, the mean-field analysis of the previous section predicts a singlet ground state that does not depend on $t$. Now we will consider quantum fluctuations near this state to obtain more accurate wave function and excitation spectra. We can rewrite Eq. (38) via Hubbard operators

$$
A_{i}^{\alpha \beta}=|\alpha\rangle_{i}\left\langle\left.\beta\right|_{i} .\right.
$$

Here $|\alpha\rangle_{i}$ and $|\beta\rangle_{i}$ belong to the set $\{S=0\},\{S=2$, $\left.S_{z}=-2, \ldots, 2\right\}$. Commutation relations between $A_{i}^{\alpha \beta}$ are very simple:

$$
\left[A_{i}^{\alpha \beta}, A_{i}^{\gamma \delta}\right]=\delta_{\beta \gamma} A_{i}^{\alpha \delta}-\delta_{\alpha \delta} A_{i}^{\gamma \beta} .
$$

Now we introduce boson operators $b_{\alpha i}^{\dagger}$ that create states with $S_{i}=2, S_{i z}=\alpha$, and $c^{\dagger}$, that creates a singlet on $i$ th site. Our physical subspace is smaller than generic Fock space of these bosons and should satisfy the condition

$$
c_{i}^{\dagger} c_{i}+\sum_{\alpha} b_{i \alpha}^{\dagger} b_{i \alpha}=1
$$

on each site.

One can easily check that if we set $A^{\alpha \beta}=b_{\alpha}^{\dagger} b_{\beta}$ for spin $S=2$ states and similar substitution with $c$ bosons when one of the states is a singlet state (which we will denote as $s$ ), then commutation relations (45) are satisfied. Since for small enough $t$ only a singlet state is occupied in the mean-field approximation, we can resolve constraint (46) using an ana$\log$ of the Holstein-Primakoff representation near the $c^{\dagger} c$ $=1$ state [37], which is given by

$$
\begin{gathered}
A_{i}^{\alpha \beta}=b_{i \alpha}^{\dagger} b_{i \beta}, A_{i}^{s \alpha}=\left(1-b_{i \beta}^{\dagger} b_{i \beta}\right)^{1 / 2} b_{i \alpha}, \\
A_{i}^{s s}=1-b_{i \beta}^{\dagger} b_{i \beta}, A_{i}^{\alpha s}=b_{i \alpha}^{\dagger}\left(1-b_{i \beta}^{\dagger} b_{i \beta}\right)^{1 / 2} .
\end{gathered}
$$

Now we expand our initial Hamiltonian in terms of now independent operators $b_{i}^{\dagger}$ up to second order:

$$
\begin{aligned}
\hat{H}^{(2)}= & \sum_{\langle i j\rangle}\left(H_{\alpha \beta ; s s} b_{\alpha i}^{\dagger} b_{\beta j}^{\dagger}+H_{s s ; \alpha \beta} b_{\alpha i} b_{\beta j}+H_{\alpha s ; s \beta} b_{\alpha i}^{\dagger} b_{\beta j}\right. \\
& \left.+H_{s \alpha ; \beta s} b_{\alpha j}^{\dagger} b_{\beta i}\right)+\frac{z}{2} \sum_{i}\left[H_{\alpha s ; \beta s} b_{\alpha i}^{\dagger} b_{\beta i}\right. \\
& \left.+H_{s \alpha ; s \beta} b_{\alpha i}^{\dagger} b_{\beta i}+H_{s s ; s s}\left(1-b_{\alpha i}^{\dagger} b_{\alpha i}-b_{\alpha j}^{\dagger} b_{\alpha j}\right)\right] .
\end{aligned}
$$

Calculation of matrices $H_{\alpha \beta ; \gamma \delta}$ gives necessary matrix elements

$$
\begin{gathered}
H_{\alpha s ; \beta s}=H_{s \alpha ; s \beta}=\delta_{\alpha \beta}\left(-\frac{20}{3} \frac{t^{2}}{U_{0}}+\frac{3 U_{2}}{z}\right), \\
H_{s s ; s s}=-\frac{20}{3} \frac{t^{2}}{U_{0}}, \\
H_{\alpha s ; s \beta}=H_{s \alpha ; \beta s}=-\delta_{\alpha \beta} \frac{8}{3} \frac{t^{2}}{U_{0}}, \\
H_{\alpha \beta ; s s}=H_{s s ; \alpha \beta}=-\frac{8}{3} \frac{t^{2}}{U_{0}}\left[\begin{array}{ccccc}
0 & 0 & 0 & 0 & 1 \\
0 & 0 & 0 & -1 & 0 \\
0 & 0 & 1 & 0 & 0 \\
0 & -1 & 0 & 0 & 0 \\
1 & 0 & 0 & 0 & 0
\end{array}\right] .
\end{gathered}
$$

We rewrite our Hamiltonian in terms of Fourier transforms of $b_{\alpha k}^{\dagger}, b_{\alpha k}$ :

$$
\begin{aligned}
\hat{H}^{(2)}= & E_{0}+\frac{z}{2} \sum_{k} \gamma_{k}\left(H_{\alpha \beta ; s s} b_{\alpha k}^{\dagger} b_{\beta-k}^{\dagger}+H_{s s ; \alpha \beta} b_{\alpha k} b_{\beta-k}\right. \\
& \left.+H_{\alpha s ; s \beta} b_{\alpha k}^{\dagger} b_{\beta k}+H_{s \alpha ; \beta s} b_{\alpha k}^{\dagger} b_{\beta k}\right) \\
& +2\left(H_{s \alpha ; s \alpha}-H_{s s ; s s}\right) b_{\alpha k}^{\dagger} b_{\alpha k}
\end{aligned}
$$

where $E_{0}$ is classical energy and 


$$
\gamma_{k}=\frac{1}{z} \sum_{e_{\mu}} e^{i \mathbf{k} \cdot \mathbf{e}_{\mu}}
$$

Now we will use canonical Bogoliubov transformations to diagonalize this Hamiltonian. Since most of the terms are diagonal in $\alpha, \beta$ subspace, it is easy to see that required transformation mixes operators $\left\{b_{0 k}^{\dagger}, b_{0-k}\right\},\left\{b_{1 k}^{\dagger}, b_{-1-k}\right\}$, $\left\{b_{-1 k}^{\dagger}, b_{1-k}\right\},\left\{b_{2 k}^{\dagger}, b_{-2-k}\right\}$, and $\left\{b_{-2 k}^{\dagger}, b_{2-k}\right\}$.

A transformation that mixes the first pair is

$$
\begin{gathered}
b_{0 k}=\cosh \theta_{k} \beta_{0 k}+\sinh \theta_{k} \beta_{0-k}^{\dagger}, \\
b_{0-k}=\cosh \theta_{k} \beta_{0-k}+\sinh \theta_{k} \beta_{0 k}^{\dagger},
\end{gathered}
$$

and complex conjugates. Substituting this transformation into Eq. (50) and requiring that terms with $\beta_{0 k}^{\dagger} \beta_{0-k}^{\dagger}$ and $\beta_{0 k} \beta_{0-k}$ vanish, we obtain the equation for $\theta_{k}$ :

$$
\tanh 2 \theta_{k}=-\frac{g_{k}}{f_{k}}=-\frac{\frac{8}{3} \frac{z t^{2}}{U_{0}} \gamma_{k}}{3 U_{2}-\frac{8}{3} \frac{z t^{2}}{U_{0}} \gamma_{k}},
$$

where

$$
\begin{gathered}
g_{k}=\frac{8}{3} \frac{z t^{2}}{U_{0}} \gamma_{k}, \\
f_{k}=3 U_{2}-\frac{8}{3} \frac{z t^{2}}{U_{0}} \gamma_{k} .
\end{gathered}
$$

The energy of this excitation becomes

$$
E(k)=\sqrt{f_{k}^{2}-g_{k}^{2}}=\sqrt{U_{2}\left(9 U_{2}-16 \frac{z t^{2}}{U_{0}} \gamma_{k}\right)} .
$$

Equation (52) suggests that the first instability appears at $k=0$ and gives the phase boundary that agrees with the metastability line $t_{+}$found in the previous subsection. Dependence of the gap on the parameters is shown in Fig. 10. However, results of the previous subsection suggest that the phase transition is first order and takes place before the mode softening at $k=0$. The first-order transition may also be obtained with the formalism presented in this section by noting that expansion of Eq. (48) allows third-order terms $c b^{\dagger} b^{\dagger}$ + c.c.

We can use a similar analysis to discuss excitations with other spin quantum numbers. For example, excitations with $S_{z}=\{+1,-1\}$ are diagonalized by analogous Bogoliubov transformations with $\theta_{k} \rightarrow-\theta_{k}$, and excitations with $S_{z}=$ $\{+2,-2\}$ are diagonalized with transformations with the same $\theta_{k}$. As required by the spin symmetry of the singlet state, all of these excitations have the same energy.

Now we can discuss the approximations made while expanding over $b_{\alpha k}^{\dagger}, b_{\beta k}$. While transformation (48) is the exact resolution of the constraint (46), expansion to the second order adds states with higher boson occupation numbers and changes Hilbert space (this is completely analogous to usual antiferromagnet spin-wave theory). However, if a posteriori we can verify that only states with occupation numbers $n_{\alpha i}$ $=\{0,1\}$ are present in the ground state, then expansion of the constraint (46) up to second order was justified. The parameter that controls such an expansion is

$$
b_{\alpha i}^{\dagger} b_{\alpha i}=\frac{1}{N} \sum b_{\alpha k}^{\dagger} b_{\alpha k}=\int \sinh ^{2} \theta_{k} \frac{d^{d} k}{(2 \pi)^{d}} \text {. }
$$

Calculation of this quantity while the singlet state is still a global maximum for $d=3$ gives numerical values $<0.001$; therefore, our expansion is much more precise than for the Heisenberg antiferromagnet, where this quantity is not much smaller than 1 and one needs the condition $S \gg 1$ to justify the spin-wave theory.

\section{E. Spin-wave excitations in the nematic phase}

Now we will consider excitations for the states with nematic order. For the states described by Eqs. (39) - (42), there are no expectation values of the spin operators $\langle\vec{S}\rangle=0$, but there is a nematic order (27) when $\theta \neq 0$. For example, when $\vec{d}$ is pointing along $z$ we find

$$
Q_{a b}=\sin ^{2} \theta\left(\begin{array}{ccc}
1 & 0 & 0 \\
0 & 1 & 0 \\
0 & 0 & -2
\end{array}\right) .
$$

In the singlet phase, expectation values of both the spin operators are $\langle\vec{S}\rangle=0$ and $\left\langle Q_{a b}\right\rangle=0$. In the singlet phase the system has a gap to all excitations of order $U_{2}$, while nematic phases have gapless spin-wave excitations that originate from the continuous symmetry breaking. The general form of the state with minimum energy is expressed via Euler angles of order parameter $\vec{d}$ as

$$
U_{z}(\gamma) U_{y}(\beta) U_{z}(\alpha)\{0,0,1,0,0\}^{T}
$$

where $U(\gamma)$ are finite angle rotation matrices. From Eqs. (53) and (28) we can express the nematic order parameter for such a state as

$$
Q_{a b}=-3 \sin ^{2} \theta\left(d_{a} d_{b}-\frac{1}{3} \delta_{a b}\right) .
$$

Goldstone theorem tells us that low-lying modes will be fluctuations of the direction of $\vec{d}$, and there will be two degenerate modes. We can utilize the approach used in the previous subsection to consider excitations in the nematic phase. Here, we should make a generalized Holstein-Primakoff expansion near the nematic state. First, we make unitary transformation in Hilbert subspace of each site, which is given by 


$$
\left(\begin{array}{c}
|0\rangle \\
|1\rangle \\
|2\rangle \\
|3\rangle \\
|4\rangle \\
|5\rangle
\end{array}\right)=\left(\begin{array}{cccccc}
\cos \theta & 0 & 0 & \sin \theta & 0 & 0 \\
0 & 0 & 1 / \sqrt{2} & 0 & 1 / \sqrt{2} & 0 \\
0 & 0 & -1 / \sqrt{2} & 0 & 1 / \sqrt{2} & 0 \\
-\sin \theta & 0 & 0 & \cos \theta & 0 & 0 \\
0 & 1 / \sqrt{2} & 0 & 0 & 0 & 1 / \sqrt{2} \\
0 & -1 / \sqrt{2} & 0 & 0 & 0 & 1 / \sqrt{2}
\end{array}\right)\left(\begin{array}{c}
|S=0, S=0\rangle \\
\left|S=2, S_{z}=-2\right\rangle \\
\left|S=2, S_{z}=-1\right\rangle \\
\left|S=2, S_{z}=0\right\rangle \\
\left|S=2, S_{z}=1\right\rangle \\
\left|S=2, S_{z}=2\right\rangle
\end{array}\right) .
$$

Making the appropriate transformation on $H_{\alpha \beta ; \gamma \delta}$, we can write our Hamiltonian as

$$
\hat{H}=\sum_{\langle i j\rangle}|\alpha\rangle_{i}|\gamma\rangle_{j} \widetilde{\mathcal{H}}_{\alpha, \beta ; \gamma, \delta}\left\langle\beta | _ { i } \left\langle\left.\delta\right|_{j},\right.\right.
$$

where states $|\alpha\rangle-|\delta\rangle$ belong to the set $\{|0\rangle-|5\rangle\}$. After that, we proceed exactly as in the previous subsection, expanding near $|0\rangle$ state. Since dependence $\theta$ on $t^{2} /\left(U_{0} U_{2}\right)$ is determined by the minimization of the energy, linear terms in $b_{k \alpha}$ and $b_{k \alpha}^{\dagger}$ are absent. Quadratic terms have exactly the same form as in Eq. (49), and all matrices become diagonal due to the proper basis choice (54). Now we can use Bogoliubov transformation to diagonalize the quadratic part. For excitations to states $|1\rangle$ and $|2\rangle$, we obtain energy dependence

$$
\begin{aligned}
E_{1}^{2}(k)= & E_{2}^{2}(k)=\frac{1}{36}\left\{-16 \gamma_{k}^{2} \frac{z^{2} t^{4}}{U_{0}^{2}}(4 \cos 2 \theta+\sqrt{2} \sin 2 \theta)^{2}\right. \\
& +\left[9 \frac{z t^{2}}{U_{0}}-18 \gamma_{k} \frac{z t^{2}}{U_{0}}+9 U_{2}+\left(2\left(\gamma_{k}-1\right) \frac{z t^{2}}{U_{0}}\right.\right. \\
& \left.+9 U_{2}\right) \cos 2 \theta-7 \frac{z t^{2}}{U_{0}} \cos 4 \theta \\
& \left.\left.+4 \sqrt{2}\left(1-\gamma_{k}\right) \frac{z t^{2}}{U_{0}} \sin 2 \theta-4 \sqrt{2} \frac{z t^{2}}{U_{0}} \sin 4 \theta\right]^{2}\right\},
\end{aligned}
$$

where $\gamma_{k}$ was defined in Eq. (51), and dependence of $\theta$ on $z t^{2} /\left(U_{0} U_{2}\right)$ is shown in Fig. 8. We find that for $k=0$, energies of these excitations are zero, as expected for nematic waves from Goldstone theorem. These excitations create

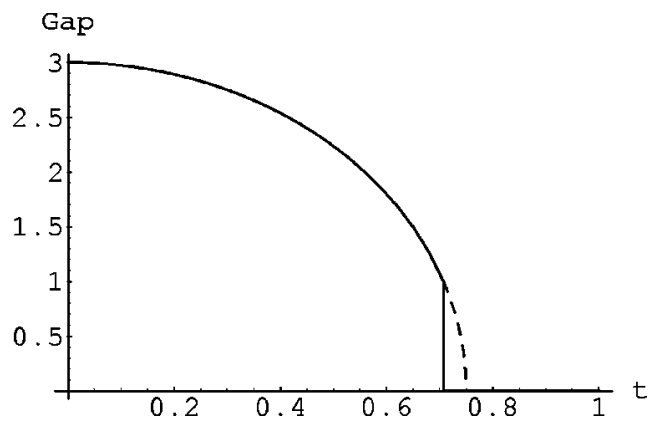

FIG. 10. Dependence of excitation gap (measured in units of $U_{2}$ ) on $t$, measured in units of $\sqrt{U_{0} U_{2} / z}$. states with $S_{z}= \pm 1$. For small $\vec{k}$, the energy of excitations depends linearly on $|\vec{k}|$, and dependence of spin-wave velocity on the parameters of the lattice is shown in Fig. 11.

Let us now consider gapped excitations for the nematic phase. Excitation to the state $|3\rangle$ corresponds to longitudinal fluctuations in the value of $\theta$, and the energy of such excitations becomes zero at $t_{-}$since at this point fluctuations of $\theta$ are not suppressed. Excitations to the states $|4\rangle$ and $|5\rangle$ correspond to the creation of $S_{z}= \pm 2$ states and they are degenerate. For all of these excitations, energies are minimized for $\vec{k}=0$. Dependence of the gap on parameters is shown in Fig. 12.

\section{F. Effects of small magnetic field}

Let us now consider the effect of a small magnetic field $\mu H \ll U_{2}$ on our system. For $U_{2}$ in the range of $\mathrm{kHz}$ (see Fig. 2 ), this corresponds to magnetic fields smaller than a $1 \mathrm{mG}$. We suppose that the field is small enough that it does not change the scattering lengths due to the energy level shifts inside of atoms. Since all atoms have the same gyromagnetic ratio, interaction with external magnetic field depends only on the total spin, and the internal structure of the states is not important. In the case of a nematically ordered insulating state, the ground-state energy does not have any contributions linear $H$ (this follows from $\langle 0|\mathbf{H} \cdot \mathbf{S}| 0\rangle=0$ ), and the second-order contribution depends on the relative orientation of the nematic-order parameter $\vec{d}$ and magnetic field $\vec{H}$. Suppose $\vec{d}$ is directing along the $z$ axis and $\vec{H}$ lies in $x, z$ plane. In second-order perturbation theory, the energy correction to the ground state is always nonpositive:

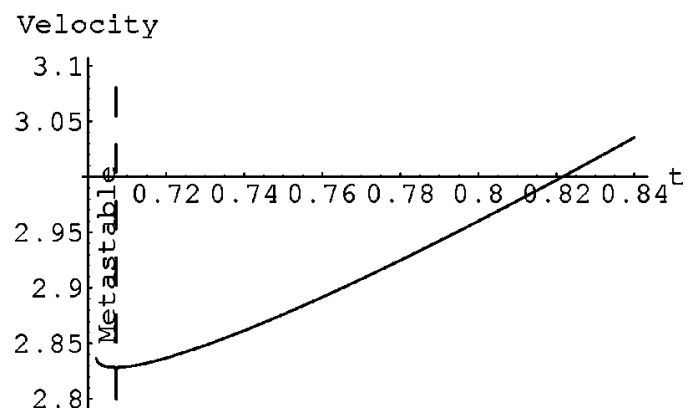

FIG. 11. Dependence of the spin-wave velocity (measured in units of $U_{2} / \sqrt{z}$ ) on $t$, measured in units of $\sqrt{U_{0} U_{2} / z}$. 


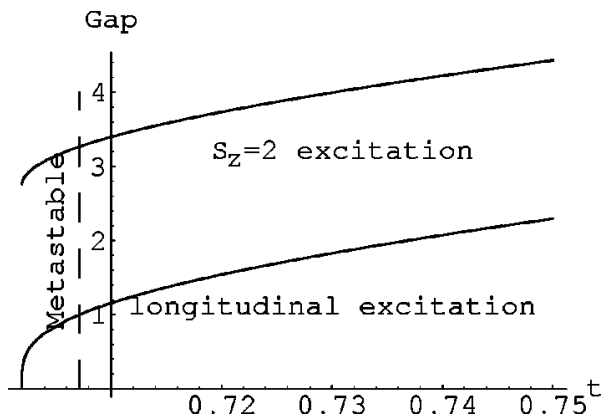

FIG. 12. Energy of the gapped excitations in the nematic phase of $N=2$ atoms per site at zero wave vector. The energy gaps and $t$ are measured in units of $U_{2}$ and $\sqrt{U_{0} U_{2} / z}$, respectively.

$$
\begin{aligned}
E^{(2)} & =-\sum_{E_{n}} \frac{\langle 0|\mu \mathbf{H} \cdot \mathbf{S}| n\rangle\langle n|\mu \mathbf{H} \cdot \mathbf{S}| 0\rangle}{E_{n}-E_{0}} \\
& =-\sum_{E_{n}}\left(\mu H_{x}\right)^{2} \frac{\left\langle 0\left|S_{x}\right| n\right\rangle\left\langle n\left|S_{x}\right| 0\right\rangle}{E_{n}-E_{0}}<0 ;
\end{aligned}
$$

this quantity is of order $-\left(\mu H_{x}\right)^{2} / t^{2}$. Since for the state with $\vec{d} \| \vec{H}$ it is again zero and the system does not benefit from magnetic field, energy is minimized when $\vec{d}$ lies in a plane perpendicular to $\vec{H}$ (this is completely analogous to an antiferromagnet). Using this property, one can distinguish a nematic phase from a singlet phase. One should apply a small magnetic field in the $z$ direction to fix the plane in which $\vec{d}$ lies, release the trap, and let the atoms fall in the gravitational field with some magnetic gradient to separate the states with different $S_{z}$ 's. Then, one should measure the quantities of each spin component. These values will have a sharp change when we cross the first-order phase transition line. Knowing how to express spin states via original boson operators, we can calculate expectation values of different spin components to be

$$
\begin{gathered}
n_{1}=n_{-1}=\frac{2}{3} \cos [\theta]^{2}+\frac{\sqrt{2}}{3} \cos [\theta] \sin [\theta]+\frac{5}{6} \sin [\theta]^{2}, \\
n_{0}=\frac{2}{3} \cos [\theta]^{2}-\frac{2 \sqrt{2}}{3} \cos [\theta] \sin [\theta]+\frac{1}{3} \sin [\theta]^{2} .
\end{gathered}
$$

Using known expressions for dependence of $\theta$ on $t$, we can make mean-field predictions on occupation numbers, shown in Fig. 13

\section{LARGE NUMBER OF PARTICLES PER SITE}

In this section we discuss the case $N \gg 1$ for both parties of $N$. We show how one can separate variables describing angular momentum and the number of particles in each well [44], and derive an effective Hamiltonian which is valid under conditions $U_{2}, N t \ll U_{0}$, which is less restrictive than in Sec. III for $N \gg 1$.

When we have $N$ spin-one bosons localized in a well in

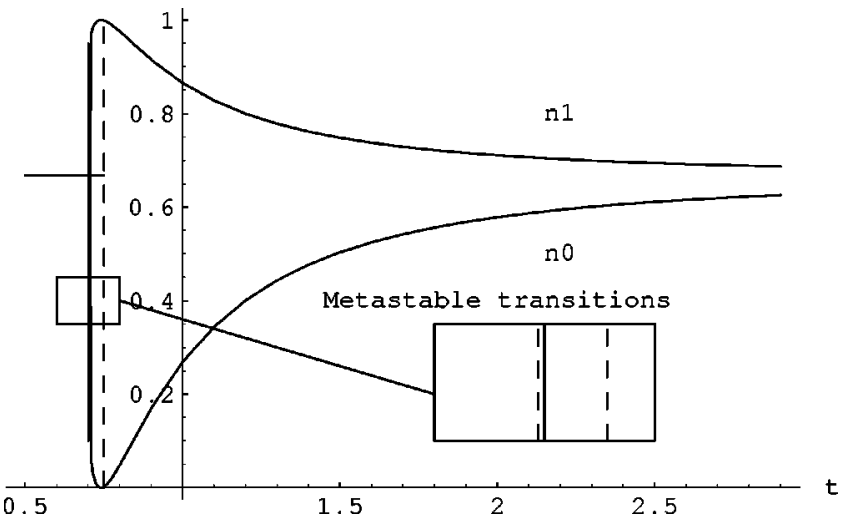

FIG. 13. Dependence of occupation numbers $n_{0}$ and $n_{1}=n_{-1}$ on $t$ for an insulating state with two bosons per site $N=2$. Tunneling $t$ is measured in units of $\sqrt{U_{0} U_{2} / z}$.

the same orbital state, their total spin may take any value that satisfies constraints

$$
\begin{gathered}
S+N=(\text { even }), \\
S \leqslant N .
\end{gathered}
$$

We define "pure condensate" wave functions as

$$
|N, \vec{n}\rangle=\frac{1}{\mathcal{N}^{\prime}}\left(n_{x} a_{x}^{\dagger}+n_{y} a_{y}^{\dagger}+n_{z} a_{z}^{\dagger}\right)^{N}|0\rangle
$$

that minimize the $U_{2}$ interaction energy at the GrossPitaevskii (mean-field) level in each given well [20]. Here $\mathcal{N}=[2(N-1) !]^{1 / 2}$ is a normalization factor, which is calculated in Appendix D. Now we can construct states such as

$$
|\psi\rangle_{N}=\int_{\mathbf{n}} \psi(\vec{n})|N, \vec{n}\rangle
$$

where $\int_{\mathbf{n}}$ stands for $\int d \mathbf{n} /(4 \pi)$.

Condition (56) corresponds to the symmetry of the states (58)

$$
|N,-\vec{n}\rangle=(-)^{N}|N, \vec{n}\rangle
$$

Hence, we need to consider only wave functions that satisfy $\psi(-\vec{n})=(-)^{N} \psi(\vec{n})$.

Now we can consider how a spin rotation operator acts on the wave function $\psi(\mathbf{n})$ :

$$
\begin{aligned}
e^{-i \theta_{\alpha} S_{\alpha}}|\psi\rangle_{N} & =\int_{\mathbf{n}} \psi(\mathbf{n}) e^{-i \theta_{\alpha} S_{\alpha}}|N, \mathbf{n}\rangle \\
& =\int_{\mathbf{n}^{\prime}} \psi\left(e^{i \theta_{\alpha} S_{\alpha} \mathbf{n}^{\prime}}\right)\left|N, \mathbf{n}^{\prime}\right\rangle \\
& =\int_{\mathbf{n}^{\prime}} \psi\left(n_{\gamma}+\epsilon_{\alpha \beta \gamma} \theta_{\alpha} n_{\beta}^{\prime}\right)\left|N, \mathbf{n}^{\prime}\right\rangle .
\end{aligned}
$$

Expanding the last expression for small $\theta$ we find 


$$
L_{\alpha} \psi=-i \epsilon_{\alpha \beta \gamma} n_{\beta} \frac{\partial}{\partial n_{\gamma}} \psi,
$$

where we used $L_{\alpha}$ rather than $S_{\alpha}$ to show that it acts on the wave function $\psi$. Therefore, operator $\mathbf{L}$ is an angular momentum operator for $\mathbf{n}$. If we want to construct any spin state, we should take $\psi(\mathbf{n})$ to be the usual spherical harmonic. We note that the $S=0$ result in Ref. [20] is just a special case of our general statement. The most general form of the state in a well can be expanded as

$$
\psi(\mathbf{n})=\sum_{|m| \leqslant L,} c_{L, m} Y_{L m}(\mathbf{n}),
$$

where $L$ satisfies conditions (56), (57).

At this point, what we have done is valid for all $N$, not only big ones. This representation is particularly suitable for $N \gg 1$ since in this limit states that correspond to different n's are orthogonal to each other (see Appendix D)

$$
\left\langle N, \mathbf{n}_{1} \mid N, \mathbf{n}_{2}\right\rangle=\delta_{N}\left(\mathbf{n}_{1}-\mathbf{n}_{2}\right) .
$$

The delta function is defined from the condition

$$
\int_{\mathbf{n}_{1}} \int_{\mathbf{n}_{2}} f_{N}^{1}\left(\mathbf{n}_{1}\right) f_{N}^{2}\left(\mathbf{n}_{2}\right) \delta_{N}\left(\mathbf{n}_{1}-\mathbf{n}_{2}\right)=\int_{\mathbf{n}} f_{N}^{1}(\mathbf{n}) f_{N}^{2}(\mathbf{n})
$$

for the functions that satisfy $f_{N}(-\mathbf{n})=(-)^{N} f_{N}(\mathbf{n})$.

We show in the Appendix D that

$$
\begin{gathered}
a_{\alpha}^{\dagger}|N, \mathbf{n}\rangle=(N+1)^{1 / 2} n_{\alpha}|N+1, \mathbf{n}\rangle, \\
a_{\alpha}|N, \mathbf{n}\rangle=N^{1 / 2} n_{\alpha}|N-1, \mathbf{n}\rangle
\end{gathered}
$$

after projecting into the "pure condensate" wave functions. This allows us to represent Eq. (65) as the product of two operators, which act in different spaces. For each trap we define the particle creation and annihilation operators that change the number of particles $N$ but not the direction of $\mathbf{n}$ [45]

$$
\begin{gathered}
b_{i}^{\dagger}\left|N_{i}, \mathbf{n}_{i}\right\rangle=\left(N_{i}+1\right)^{1 / 2}\left|N_{i}+1, \mathbf{n}_{i}\right\rangle, \\
b_{i}\left|N_{i}, \mathbf{n}_{i}\right\rangle=N_{i}^{1 / 2}\left|N_{i}-1, \mathbf{n}_{i}\right\rangle .
\end{gathered}
$$

The number of particles in each trap may be expressed using $b$ operators as

$$
N_{i}=b_{i}^{\dagger} b_{i} .
$$

Hamiltonian (5) can now be represented as

$$
\begin{aligned}
\mathcal{H}= & -t \sum_{\langle i j\rangle, \sigma} \mathbf{n}_{i} \cdot \mathbf{n}_{j}\left(b_{i}^{\dagger} b_{j}+b_{j}^{\dagger} b_{i}\right)-\mu \sum_{i} \hat{N}_{i} \\
& +\frac{U_{0}}{2} \sum_{i} \hat{N}_{i}\left(\hat{N}_{i}-1\right)+\frac{U_{2}}{2} \sum_{i}\left(\vec{L}_{i}^{2}-2 \hat{N}_{i}\right),
\end{aligned}
$$

where

$$
\mathbf{L}_{i}=-i \mathbf{n}_{i} \times \frac{\partial}{\partial \mathbf{n}_{i}} .
$$

Now, if we are in the Mott insulating phase, we can easily derive the effective Hamiltonian for $\psi(\mathbf{n})$. Using the secondorder perturbation theory, we find the effective Hamiltonian on the sphere to be

$$
\begin{gathered}
\mathcal{H}=\frac{U_{2}}{2} \sum_{i} \vec{L}_{i}^{2}-\frac{2 N^{2} t^{2}}{U_{0}} \sum_{i j} n_{i a} n_{i b} n_{j a} n_{j b}, \\
\psi(\mathbf{n})=(-1)^{N} \psi(-\mathbf{n}) .
\end{gathered}
$$

We note that this Hamiltonian corresponds to a lattice of quantum rotors that interact via quadrupolar moments.

\section{Mean-field solution}

Now we can find a mean field ground state of Eq. (70). We consider the case when the quadrupolar interaction term is much bigger than the kinetic term. We show that in this case the ground state is a uniaxial nematic and find its energy. Comparing the energy of this state to that of a singlet, we estimate the phase boundary for nematic-singlet transition for even $N$.

Our general mean-field ansatz has the form

$$
|\Psi\rangle=\prod_{i} \Psi\left(\theta_{i}, \varphi_{i}\right), \int_{\mathbf{n}}|\Psi(\theta, \varphi)|^{2}=1 .
$$

The expectation value of Eq. (70) per well over the wave function (71) equals

$$
\frac{U_{2}}{2}\left\langle\vec{L}_{i}^{2}\right\rangle-J\left\langle n_{\alpha} n_{\beta}\right\rangle\left\langle n_{\beta} n_{\alpha}\right\rangle,
$$

where

$$
\begin{gathered}
J=\frac{z N^{2} t^{2}}{U_{0}}, \\
\vec{L}_{i}^{2}=-\frac{1}{\sin \theta} \frac{\partial}{\partial \theta}\left(\sin \theta \frac{\partial}{\partial \theta}\right)-\frac{1}{\sin ^{2} \theta} \frac{\partial^{2}}{\partial \varphi^{2}} .
\end{gathered}
$$

As in the case of $N=2$, all of the states that can be transformed into each other by global rotation have the same energy. Therefore, we can impose three additional conditions. The best choice is to require symmetric real matrix $\left\langle n_{i} n_{j}\right\rangle$ to be diagonal and to choose $\left\langle n_{z}^{2}\right\rangle$ to be the biggest eigenvalue. In such a gauge interaction, the term becomes $-J\left(\left\langle n_{x}^{2}\right\rangle^{2}+\left\langle n_{y}^{2}\right\rangle^{2}+\left\langle n_{z}^{2}\right\rangle^{2}\right)$. Since we have the extra constraint $\left\langle n_{x}^{2}\right\rangle+\left\langle n_{y}^{2}\right\rangle+\left\langle n_{z}^{2}\right\rangle=1$, it is now obvious that interaction energy is minimized when

$$
\left\langle n_{z}^{2}\right\rangle=\cos ^{2} \theta \rightarrow 1 .
$$

However, states with $\sin ^{2} \theta \rightarrow 0$ have higher angular moments, and the ground state is determined by the competition of 
these two factors. We write mean-field Gross-Pitaevskii equations to determine the ground state

$$
\begin{gathered}
\left\{-\frac{U_{2}}{2} \frac{1}{\sin \theta} \frac{\partial}{\partial \theta}\left(\sin \theta \frac{\partial}{\partial \theta}\right)-\frac{1}{\sin ^{2} \theta} \frac{\partial^{2}}{\partial \varphi^{2}}-2 J\left(n_{x}^{2}\left\langle n_{x}^{2}\right\rangle\right.\right. \\
\left.\left.+n_{y}^{2}\left\langle n_{y}^{2}\right\rangle+n_{z}^{2}\left\langle n_{z}^{2}\right\rangle\right)\right\} \Psi(\theta, \varphi)=\lambda \Psi(\theta, \varphi),
\end{gathered}
$$

where $\lambda$ is Lagrange multiplier. Now we consider the case $J \gg U_{2}$. In this case interaction energy dominates and we expect $\sin ^{2} \theta \rightarrow 0$ and, therefore, wave function becomes localized near $z$ and $-z$ directions. We can solve the problem by expanding only near $\theta=0$ and then taking an (anti)symmetric combination to satisfy Eq. (70). It is obvious that if we expand the kinetic part of Eq. (74) up to the first nonvanishing order in $\theta$, then we will get a two-dimensional Laplace operator $\Delta_{n_{x}, n_{y}}$, and our problem becomes equivalent to a harmonic oscillator. Effective parameters are expressed as

$$
m=\frac{1}{U_{2}}, \omega_{x, y}^{2}=4 J U_{2}\left\langle n_{z}^{2}-n_{x, y}^{2}\right\rangle .
$$

Since we have already neglected higher order terms in $\left\langle\theta^{2}\right\rangle$ while obtaining a harmonic Hamiltonian from Eq. (74), with the same accuracy we can set $\omega_{x}=\omega_{y}=\sqrt{4 J U_{2}}$ in Eq. (75), i.e., the ground state is a uniaxial nematic. Since we know wave functions, we can calculate the expectation value of energy. We will use the fact that for a harmonic oscillator the expectation value of kinetic energy is the same as that of potential energy. The energy of the ground state becomes

$$
E=\frac{3}{8}\left(\omega_{x}+\omega_{y}\right)-J\left\langle n_{z}^{2}\right\rangle
$$

Quantum fluctuations of the direction of $\mathbf{n}$ equal

$$
\left\langle n_{x}^{2}\right\rangle=\left\langle n_{y}^{2}\right\rangle=\frac{U_{2}}{2 \omega}=\frac{1}{4} \sqrt{\frac{U_{2}}{J}}
$$

and the expectation value of the ground-state energy is

$$
E=\frac{3}{4} \omega+\frac{J U_{2}}{\omega}-J=2 \sqrt{U_{2} J}-J .
$$

Symmetrization or antisymmetrization of the wave function introduces exponentially small shifts in energy, so in the limit $J \gg U_{2}$ energy does not feel the parity of $N$. Though in this subsection we explicitly started from variational ansatz (71), now we can justify it in the limit $J \gg U_{2}$ since in this case quantum fluctuations of the direction of $\hat{\mathbf{n}}$ are small and given by Eq. (76).

From Sec. III we know that for small nonzero $t$ there is a uniaxial nematic state for odd $N$. Since in the opposite limit there is also a nematic state, we expect that for all $N \gg 1, N$ $=2 n+1$ the insulating state will be nematic. For the case of even $N$, there is always a singlet state in which mean-field energy equals $-J / 3$. Comparing this energy with Eq. (77), we can estimate the first-order transition point as $U_{2}=J / 9$. At this point

$$
\left\langle n_{x}^{2}\right\rangle=1 / 12
$$

so we expect our expansion to be valid.

\section{GLOBAL PHASE DIAGRAM}

In the earlier sections we have established spin structure of insulating phases of $S=1$ bosons in the optical lattice in various limits. Here we summarize our arguments and discuss implications of our results for the global phase diagram.

\section{A. Two- and three-dimensional lattices}

In two- and three-dimensional lattices, insulating states with one atom per site are nematic as long as the perturbation theory approach in $t / U_{0}$ remains valid, as was shown in Sec. III. For an arbitrary odd number of particles per site $N$ and in the limit of small tunneling $(N t)^{2} / U_{0} \ll U_{2}$, the nematic order in the ground state was also established in Sec. III. For large, odd $N$, the nematic order in the ground state can be proven when $(N t)^{2} / U_{0}$ becomes larger than $U_{2}$ (but still smaller than $U_{0}$ ), as was demonstrated in Sec. V. It is also natural to expect that the superfluid polar phase develops from the nematic insulator (both states break spin rotational symmetry without breaking the time reversal symmetry), so we expect the nematic order even when $N t / U_{0}$ is not small and the system is close to the superfluid-insulator transition. In all cases we find that insulating phases with an odd number of particles per site are nematic.

In the case of two particles per site, the results of Sec. IV establish that for small enough $U_{2} / U_{0}$ there is a first-order transition between the spin-singlet phase (for small $t$ ) and the spin-nematic phase (for larger $t$ ) at $z t_{c}^{2} / U_{0} U_{2}=0.5(z$ is the coordination number of the lattice). Analogously, for large, even $N$, results of Sec. V show that the singlet insulating ground state goes into spin nematic at $z N^{2} t_{c}^{2} / U_{0} U_{2}=9$. Since for small enough $U_{2} / U_{0}$ we expect nematic spin order close to the SI transition into the polar superfluid phase, we propose that in this case insulating phases with an even number of particles per site are either singlet or nematic with the first-order transition at some critical value of tunneling $t_{c}$.

In all of our earlier discussions, we assumed that Mott insulating lobes for even fillings are big enough to have a transition into the nematic phase before superfluidity sets in. This assumption is controlled by the smallness of the ratio $U_{2} / U_{0}$. Here we will discuss the superfluid-insulator phase boundaries and estimate how small $U_{2} / U_{0}$ should be for the singlet-nematic transition to lie inside the Mott phase.

Assuming a transition from the spin-singlet insulating phase, the mean-field calculation of the superfluid-insulator phase boundary was given in Ref. [46]. The analysis presented in this paper shows that the critical value of tunneling, after which the Mott phase does not exist, is given by

$$
\frac{U_{0}+2 U_{2}}{z t_{\mathrm{SI}}}=\frac{1}{3}\left(2 N+3+2 \sqrt{N^{2}+3 N}\right) .
$$




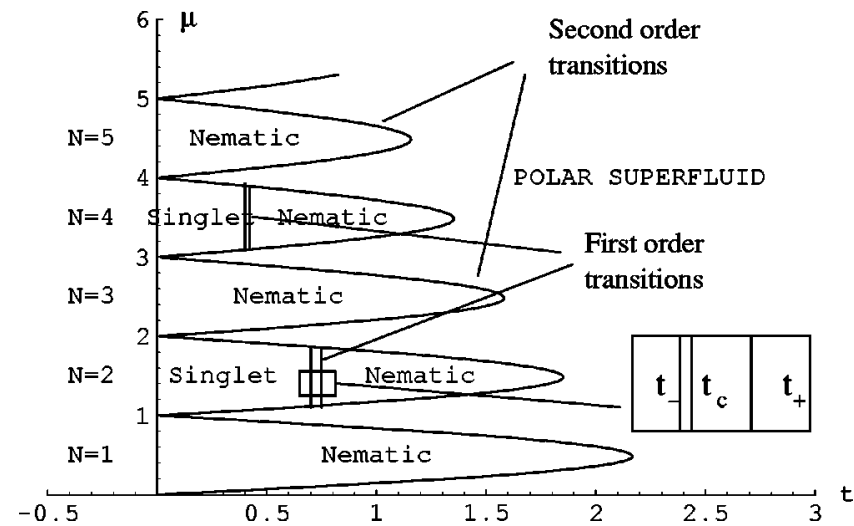

FIG. 14. Global phase diagram for $S=1$ bosons in 2D and 3D optical lattice for $z U_{2} / U_{0}<0.1$. Mean-field analysis of the superfluid to insulator transition was done in Ref. [46]. In this work we concentrated on discussing the spin structure of the insulating lobes. A singlet-nematic first-order phase transition for $N=2$ takes place for $z t^{2} / U_{0} U_{2}=0.5$ ( $z$ is the coordination number of the lattice). For large even $N$, a singlet-nematic phase transition occurs at $z N^{2} t^{2} / U_{0} U_{2}=9 . t_{c}$ marks the actual first-order phase transition and $t_{-}$and $t_{+}$are the limits of metastability. Note that the system may also have fragmented superfluid phases for small $t$ that are not shown here [27].

We will use this critical value $t_{\mathrm{SI}}$ as an estimate of the superfluid-insulator transition. For $N=2$, the singlet-nematic phase transition takes place at $z t_{c}^{2} / U_{0} U_{2}=0.5$. The condition $t_{c}<t_{\text {SI }}$ for $N=2$ is satisfied, if

$$
\frac{z U_{2}}{U_{0}}<0.1
$$

For the case $N \gg 1$ the requirement of $t_{c}<t_{\mathrm{SI}}$ becomes even more restrictive, namely,

$$
\frac{z U_{2}}{U_{0}}<0.01
$$

One can see that depending on the exact value of $z U_{2} / U_{0}$, there are different possibilities for Mott lobes with an even number of particles. When $z U_{2} / U_{0}<0.01$, all insulating phases with even filling factors are spin singlet for small tunneling and spin nematic for larger tunneling. For 0.01 ${ }_{z} U_{2} / U_{0}<0.1$, insulating phases with small, even filling factors have both singlet and nematic regimes, but insulating states with sufficiently large even fillings have only a singlet phase. Finally, for $0.1<_{z} U_{2} / U_{0}$, all insulating phases with even filling factors are in the spin-singlet state. In Figs. 14 and 15 we combine these results with the schematic representation of the SI transitions to obtain the global phase diagram.

\section{B. One-dimensional lattices}

For one-dimensional lattices we established that when $N^{2} t^{2} / U_{0} \ll U_{2}$ the system will be in a uniform singlet phase for even fillings and in a dimerized singlet phase for odd fillings (when there is only one atom per site the dimerized

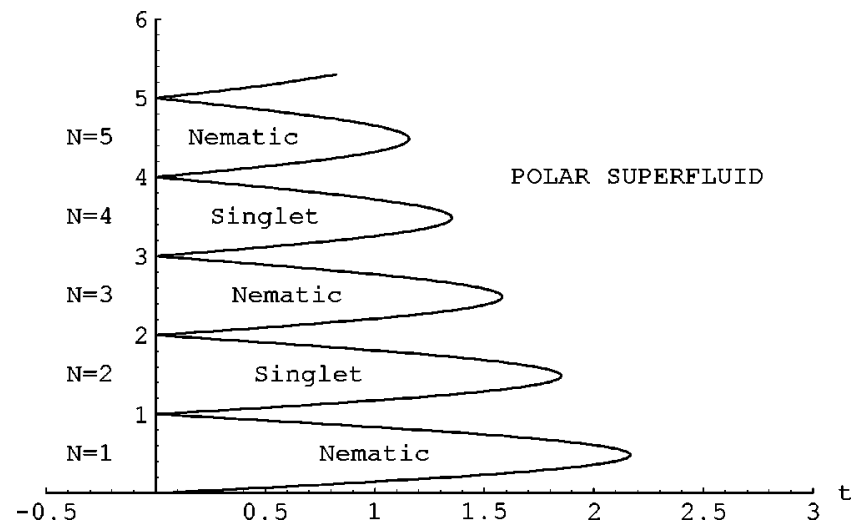

FIG. 15. Global phase diagram for $S=1$ bosons in 2D and 3D optical lattice, for $z U_{2} / U_{0}>0.1$. A superfluid-insulator transition for even filling factors takes place before singlet-nematic transition.

phase has been verified in the regime $\left.t \ll U_{0}\right)$. The nature of magnetic order close to the tips of the insulating lobes (when the perturbation theory in $t$ is not applicable) is less clear. However, we expect that the phase diagram for the onedimensional lattice is qualitatively similar to two- and threedimensional cases with one important difference: instead of the nematic phase, we have dimerized singlet states. This will be discussed in future publications.

\section{DETECTION OF SPIN ORDER IN INSULATING PHASES}

Now we discuss two approaches to detect the singletnematic phase transition for $S=1$ bosons in an optical lattice. One way of detecting such a transition has already been noted in Sec. IV F, where we proposed to introduce an easy plane for nematic order by applying a small magnetic field, then releasing the trap and measuring the number of particles of different spin components. Spatial separation of different spin components can be achieved by applying magnetic field gradients during the free fall of the atoms. For the case of $N=2$, with a small magnetic field applied in the $z$ direction, expectation values of $n\left(S_{z}=0\right)$ and $n\left(S_{z}=1\right)=n\left(S_{z}=-1\right)$ have been calculated and are shown in Fig. 13. Since the phase transition is of first order, there is a sharp change which can be measured experimentally. We note that the $N$ $=2$ case also have a particular experimental advantage over other filling factors due to the absence of three particle losses and the least restrictive condition on $U_{2} / U_{0}$ for observation of the singlet-nematic transition.

The second approach to experimental detection of singlet and nematic insulating phases relies on the measurement of excitation spectra. As discussed in Secs. IV D and IV E, the singlet phase has a nonzero gap to all excitations, whereas the nematic phase has gapless spin-wave excitations. To measure the excitation spectra, we propose using Bragg spectroscopy (see in Fig. 16), which was used successfully to identify soundlike Bogoliubov excitations in condensates of spinless particles [47]. In such experiments the optical lattice should be illuminated by two laser beams with wave vectors $\mathbf{k}_{\mathbf{1}}$ and $\mathbf{k}_{\mathbf{2}}$ and a frequency difference $\omega$, which is much smaller than their detuning from an atomic resonance. The 


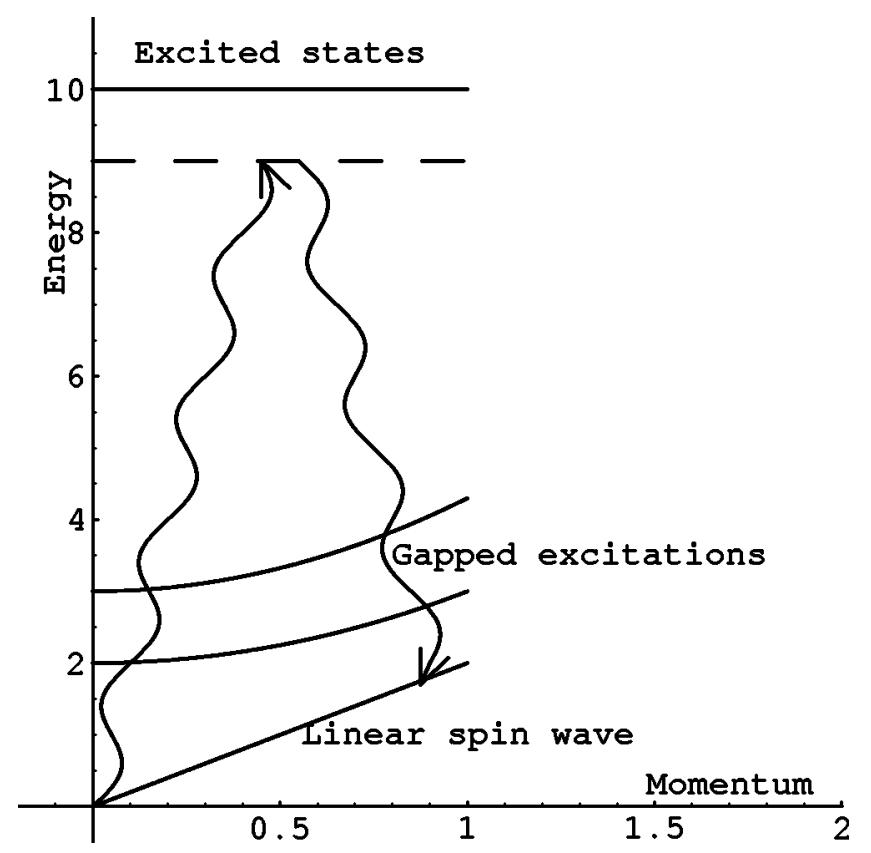

FIG. 16. Probing dispersion relation using Bragg scattering.

intersecting beams create a periodic traveling intensity modulation that creates external potential due to an ac Stark effect of the form $V_{\alpha \beta} \cos (\mathbf{q} \cdot \mathbf{r}-\omega t)$. Here we introduce spin indices for the external potential since the ac Stark effect may introduce mixing between different $S_{z}$ components. A response of the system to such a potential may be calculated using Fermi's golden rule. Interaction is expressed in secondquantized notations such as $V_{\alpha \beta} / 2\left[\hat{\boldsymbol{\rho}}_{\alpha \beta}^{\dagger}(\mathbf{q}) e^{-i \omega t}+\hat{\boldsymbol{\rho}}_{\alpha \beta}^{\dagger}\right.$ $\left.(-\mathbf{q}) e^{i \omega t}\right]$, where $\hat{\boldsymbol{\rho}}_{\alpha \beta}^{\dagger}=\Sigma_{k} a_{\alpha k+q}^{\dagger} a_{\beta k}$. The scattering rate is given by

$$
\frac{2 \pi}{\hbar} \sum_{f}\left|\left\langle f\left|V_{\alpha \beta} \hat{\rho}_{\alpha \beta}^{\dagger}\right| g\right\rangle\right|^{2} \delta\left(\hbar \omega-E_{f}+E_{g}\right) .
$$

If the resonance state is far detuned from the excited states, then $V_{\alpha \beta}$ has the form $V \delta_{\alpha \beta}$, and couples only to the total number of particles in each well and does not feel internal spin structure. Low-lying excitations in insulating phases do not change the number of particles on individual sites, so the $V \delta_{\alpha \beta}$ interaction will not produce any Bragg peaks for lowlying excitations. Therefore, it is necessary for detection that $V_{\alpha \beta}$ deviate from $V \delta_{\alpha \beta}$, which can be achieved by making detuning comparable to level spacing of fine and hyperfine components. From Sec. IV E we know that for $N=2$, nematic spin-wave excitations correspond to $S_{z}= \pm 1$, longitudinal excitation corresponds to $S_{z}=0$, and there are also gapped excitations with $S_{z}= \pm 2$. Since the nematic state has $S_{z}=0$, it is necessary to have nonzero $V_{0, \pm 1}, V_{0,0}$, and $V_{ \pm 1, \mp 1}$ to observe each kind of these excitations. In Fig. 17 we show dependence of $N=2$ peak positions on $t$ for fixed q.

Finally, we consider the effect of inhomogeneous trapping potential. When this local trapping potential $\varphi_{i}$ varies smoothly from site to site, it is not the chemical potential $\mu_{i}$ which is fixed across the trap, but the sum $\varphi_{i}+\mu_{i}$. There-

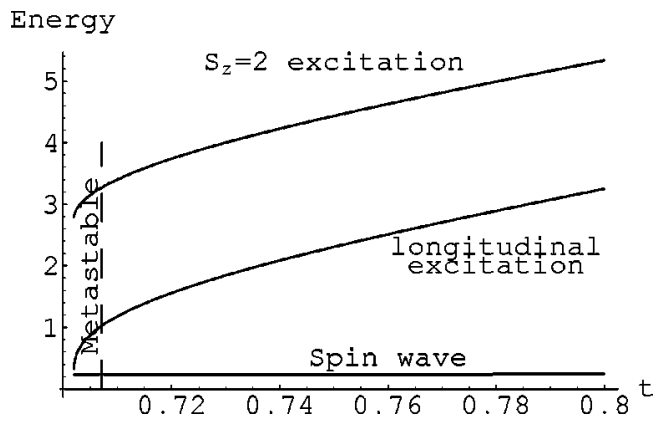

FIG. 17. Positions of Bragg peaks for $|\mathbf{q}|=0.02, z=6$ in the nematic phase with $N=2$ atoms per site. Energy is measured in units of $U_{2}$ and $t$ is measured in units of $\sqrt{U_{0} U_{2} / z}$.

fore, if $\varphi_{i}$ varies considerably, we will have insulating regions with different occupation numbers as well as regions with superfluid order, all in the same trap, as was discussed in Ref. [16] for the case of spinless atoms. Therefore, Bragg scattering experiments for fixed $\mathbf{q}$ will exhibit resonances coming from the regions of the lattice at different filling factors. The relative intensity of these resonances will be determined by the relative number of particles in each region. Interpretation of Bragg experiments will be easier if the trapping potential is not harmonic, but has sharp borders, so the whole system has essentially the same density.

\section{CONCLUSIONS}

In summary, we have considered Mott insulating phases of spin-1 atoms with antiferromagnetic interactions in optical lattices. In the experimentally interesting limit $U_{2} \ll U_{0}$, and deep inside the Mott phases $N t \ll U_{0}$ ( $N$ is the filling factor), we performed detailed calculations for the following cases: (i) odd number of particles per site and $(N t)^{2} / U_{0} \ll U_{2}$, (ii) two particles per site and an arbitrary ratio of $t^{2} / U_{0}$ and $U_{2}$, and (iii) large number of particles per site $N \gg 1$ with an arbitrary ratio of $(N t)^{2} / U_{0}$ and $U_{2}$. Based on this analysis we argued that in two- and three-dimensional lattices insulating phases with an odd number of particles per site are always nematic. For an even number of particles per site, there is either a spin singlet phase or a first-order phase transition between spin singlet and nematic phases controlled by the depth of the optical lattice. The resulting global phase diagrams are shown in Figs. 15 and 14. We have considered excitations for singlet and nematic phases and have reviewed the effects of small magnetic field. For one-dimensional lattices we have found dimerized singlet phases for insulating states with odd fillings. We also discussed different experimental techniques to identify the proposed phases.

\section{ACKNOWLEDGMENTS}

We thank E. Altman, D. Haldane, W. Hofstetter, D. Podolsky, S. Sachdev, A. Sorensen, D.W. Wang, and F. Zhou for useful discussions. This work was partially supported by the NSF Career Award No. DMR-0132874, PHY-0134776, and by the Sloan and Packard Foundations. When this work neared completion, we learned that similar results have been obtained by M. Snoek and F. Zhou [48]. 


\section{APPENDIX A: DERIVATION OF THE EFFECTIVE MAGNETIC HAMILTONIAN FOR AN INSULATING STATE WITH ODD NUMBER OF ATOMS}

To be able to derive the $J_{0}, J_{1}, J_{2}$ dependence on $n$, we should know how to write down explicitly, in terms of creation and annihilation operators, all of the states that we are interested in. To do this we introduce the singlet pair creation operator (summation over repeated indices is presumed over $\{x, y, z\})$

$$
\theta^{\dagger}=a_{p}^{\dagger} a_{p}^{\dagger}=\left(a_{0}^{\dagger}\right)^{2}-2 a_{-}^{\dagger} a_{+}^{\dagger},
$$

which has the following commutation relations:

$$
\begin{gathered}
{\left[a_{p}, \theta^{\dagger}\right]=2 a_{p}^{\dagger}, \quad\left[a_{0}, \theta^{\dagger}\right]=2 a_{0}^{\dagger}, \quad\left[a_{ \pm}, \theta^{\dagger}\right]=-2 a_{\mp}^{\dagger},} \\
{\left[a^{\dagger}, \theta^{\dagger}\right]=0,\left[S_{a}, \theta^{\dagger}\right]=0,\left[\vec{S}^{2}, \theta^{\dagger}\right]=0 .}
\end{gathered}
$$

Since $\theta^{\dagger}$ commutes with spin operators, it does not change total spin and spin components but just adds two electrons. Therefore, we can construct unnormalized spin states for arbitrary $n$ in the following way: first, write down a state with necessary spin for a small number of particles; second, apply $\theta^{\dagger}$ as many times as needed to get the desired number of particles.

Using this procedure we obtain that states

$$
a_{+}^{\dagger}\left(\theta^{\dagger}\right)^{n}|0\rangle, \quad a_{-}^{\dagger}\left(\theta^{\dagger}\right)^{n}|0\rangle, \quad a_{0}^{\dagger}\left(\theta^{\dagger}\right)^{n}|0\rangle
$$

belong to $S=1$ low-energy subspace and are orthogonal since they are different eigenvectors of $S_{z}$. Making an orthogonal transformation that leads to the $\left\{a_{x}^{\dagger}, a_{y}^{\dagger}, a_{z}^{\dagger}\right\}$ basis, we can write three orthonormal states with $S=1$ as

$$
|S=1, p, 2 n+1\rangle=\frac{1}{\sqrt{f(n ; 1)}} a_{p}^{\dagger}\left(\theta^{\dagger}\right)^{n}|0\rangle,
$$

where normalization factor

$$
f(n ; s)=s ! n ! 2^{n} \frac{(2 n+2 s+1) ! !}{(2 s+1) ! !}
$$

was calculated in Ref. [21]. In our calculation later we will need more general normalization factors, so first we will derive the way to normalize our spin states.

\section{Normalization of the states}

We will be interested in normalization of the states

$$
|a, b, n\rangle=a_{a}^{\dagger} a_{b}^{\dagger}\left(\theta^{\dagger}\right)^{n}|0\rangle
$$

and calculation of

$f(a, b, p, q, n)=\langle a, b, n \mid p, q, n\rangle=\left\langle 0\left|(\theta)^{n} a_{a} a_{b} a_{p}^{\dagger} a_{q}^{\dagger}\left(\theta^{\dagger}\right)^{n}\right| 0\right\rangle$, where $a, b, p, q \in\{x, y, z\}$. Let us consider a coherent state

$$
e^{a_{x}^{\dagger} x_{1}+a_{y}^{\dagger} x_{2}+a_{z}^{\dagger} x_{3}}|0\rangle
$$

We observe that this state is a linear combination of Fock states, with the coefficients being polynomials in $\left\{x_{1}, x_{2}, x_{3}\right\}$. To extract the weight of the state $|a, b, n\rangle$, we need to calculate the quantity

$$
|a, b, n\rangle=\left[T_{a, b}^{n}(x) e^{a_{x}^{\dagger} x_{1}+a_{y}^{\dagger} x_{2}+a_{z}^{\dagger} x_{3}}|0\rangle\right]_{\mathbf{x}=\mathbf{0}},
$$

where

$$
T_{a, b}^{n}(x)=\nabla_{x_{a}} \nabla_{x_{b}}\left(\Delta_{x}\right)^{n}
$$

We use the normalization condition for coherent states (see, e.g., Ref. [49])

$$
\left\langle 0\left|e^{a_{x} y_{1}+a_{y} y_{2}+a_{z} y_{3}} e^{a_{x}^{\dagger} x_{1}+a_{y}^{\dagger} x_{2}+a_{z}^{\dagger} x_{3}}\right| 0\right\rangle=e^{x_{1} y_{1}+x_{2} y_{2}+x_{3} y_{3}}
$$

to calculate

$$
\begin{aligned}
& {\left.\left[T_{a, b}^{n}(x) T_{p, q}^{n}(y) e^{x_{1} y_{1}+x_{2} y_{2}+x_{3} y_{3}}\right]\right|_{\mathbf{x}=\mathbf{0}, \mathbf{y}=\mathbf{0}}} \\
& \quad=T_{a, b}^{n}(x) T_{p, q}^{n}(y) \frac{\left(x_{1} y_{1}+x_{2} y_{2}+x_{3} y_{3}\right)^{2 n+2}}{(2 n+2) !} .
\end{aligned}
$$

We can expand $T_{a, b}^{n}(x) T_{p, q}^{n}(y)$ using the extended Newton binomial formula

$$
\begin{aligned}
& T_{a, b}^{n}(x) T_{p, q}^{n}(y) \\
& =\sum_{n_{1}+n_{2}+n_{3}=n} \frac{n !}{n_{1} ! n_{2} ! n_{3} !} \nabla_{x_{1}}^{2 n_{1}} \nabla_{x_{2}}^{2 n_{2}} \nabla_{x_{3}}^{2 n_{3}} \nabla_{x_{a}} \nabla_{x_{b}} \\
& \quad \times \sum_{m_{1}+m_{2}+m_{3}=n} \frac{n !}{m_{1} ! m_{2} ! m_{3} !} \nabla_{y_{1}}^{2 m_{1}} \nabla_{y_{2}}^{2 m_{2}} \nabla_{y_{3}}^{2 m_{3}} \nabla_{y_{p}} \nabla_{y_{q}} .
\end{aligned}
$$

Let us first consider the case when sets $\{a, b\}$ and $\{p, q\}$ coincide. We have two essentially different cases: $a=b$ and $a \neq b$. Without loss of generality, suppose for the first case $a=b=p=q=x$. Then,

$$
\begin{aligned}
& \langle a, a, n \mid a, a, n\rangle \\
& =\sum_{n_{1}+n_{2}+n_{3}=n, l_{1}+l_{2}+l_{3}=2 n+2}\left(\frac{n !}{n_{1} ! n_{2} ! n_{3} !}\right)^{2} \frac{1}{l_{1} ! l_{2} ! l_{3} !} \\
& \quad \times\left(\nabla_{x_{1}} \nabla_{y_{1}}\right)^{2 n_{1}+2}\left(\nabla_{x_{2}} \nabla_{y_{2}}\right)^{2 n_{2}}\left(\nabla_{x_{3}} \nabla_{y_{3}}\right)^{2 n_{3}}\left(x_{1} y_{1}\right)^{l_{1}} \\
& \quad \times\left(x_{2} y_{2}\right)^{l_{2}}\left(x_{3} y_{3}\right)^{l_{3}} \\
& =\sum_{n_{1}+n_{2}+n_{3}=n}\left(\frac{n !}{n_{1} ! n_{2} ! n_{3} !}\right)^{2}\left(2 n_{1}+2\right) !\left(2 n_{2}\right) !\left(2 n_{3}\right) ! .
\end{aligned}
$$

This double sum can be calculated; the answer is

$$
f(x, x, x, x, n)=\frac{2}{15}(3+2 n)(5+3 n)(2 n+1) ! .
$$

For the case of different indices, the normalization is 


$$
\begin{aligned}
& f(x, y, x, y, n)=\sum_{n_{1}+n_{2}+n_{3}=n, l_{1}+l_{2}+l_{3}=2 n+2}\left(\frac{n !}{n_{1} ! n_{2} ! n_{3} !}\right)^{2} \\
& \times \frac{1}{l_{1} ! l_{2} ! l_{3} !}\left(\nabla_{x_{1}} \nabla_{y_{1}}\right)^{2 n_{1}+1}\left(\nabla_{x_{2}} \nabla_{y_{2}}\right)^{2 n_{2}+1} \\
& \times\left(\nabla_{x_{3}} \nabla_{y_{3}}\right)^{2 n_{3}}\left(x_{1} y_{1}\right)^{l_{1}}\left(x_{2} y_{2}\right)^{l_{2}}\left(x_{3} y_{3}\right)^{l_{3}} \\
& \times \sum_{n_{1}+n_{2}+n_{3}=n}\left(\frac{n !}{n_{1} ! n_{2} ! n_{3} !}\right)^{2} \\
& \times\left(2 n_{1}+1\right) !\left(2 n_{2}+1\right) !\left(2 n_{3}\right) \text { ! } \\
& =\frac{1}{15}(3+2 n)(5+2 n)(2 n+1) ! \text {. }
\end{aligned}
$$

Now let us consider the case when $\{a, b\}$ and $\{p, q\}$ do not coincide. There are four essentially different cases

$$
(x, y, x, z), \quad(x, x, y, z), \quad(x, x, x, y), \quad(x, x, y, y) .
$$

All other normalizations can be obtained from these by proper permutation of indices. In the first three cases, overlap is zero since (say, for the first case) a nonzero value comes from the term that satisfies the conditions

$$
2 n_{1}+1=2 m_{1}+1,2 n_{2}+1=2 m_{2}, 2 n_{3}=2 m_{3}+1
$$

which does not have integer solutions. However, in the fourth case, overlap of the states is not zero: it comes from the terms obeying

$$
n_{1}+1=m_{1}, n_{2}=m_{2}+1, n_{3}=m_{3} \text {. }
$$

We can calculate this quantity analogously to the previous calculation, but we can use another trick:

$$
\begin{aligned}
\left\langle 0\left|(\theta)^{n+1}\left(\theta^{\dagger}\right)^{n+1}\right| 0\right\rangle= & 3\left\langle 0\left|(\theta)^{n} a_{x} a_{x} a_{x}^{\dagger} a_{x}^{\dagger}\left(\theta^{\dagger}\right)^{n}\right| 0\right\rangle \\
& +6\left\langle 0\left|(\theta)^{n} a_{x} a_{x} a_{y}^{\dagger} a_{y}^{\dagger}\left(\theta^{\dagger}\right)^{n}\right| 0\right\rangle .
\end{aligned}
$$

Therefore,

$$
\begin{aligned}
f(x, x, y, y, n) \\
=\frac{1}{6}\left((2 n+3) !-3 \frac{2}{15}(3+2 n)(5+3 n)(2 n+1) !\right) \\
=\frac{2}{15} n(3+2 n)(2 n+1) !
\end{aligned}
$$

Since sometimes it will be more convenient for us to work in the $\{+,-, 0\}$ basis, let us also write down all nonzero overlaps in this basis (up to trivial permutations):

$$
\begin{aligned}
f( \pm, \pm, \pm, \pm, n) & =\left\langle 0\left|(\theta)^{n} a_{ \pm} a_{ \pm} a_{ \pm}^{\dagger} a_{ \pm}^{\dagger}\left(\theta^{\dagger}\right)^{n}\right| 0\right\rangle \\
& =\frac{2}{15}(5+2 n)(3+2 n)(2 n+1) !,
\end{aligned}
$$

$$
\begin{aligned}
f(0,0,0,0, n) & =\left\langle 0\left|(\theta)^{n} a_{0} a_{0} a_{0}^{\dagger} a_{0}^{\dagger}\left(\theta^{\dagger}\right)^{n}\right| 0\right\rangle \\
= & \frac{2}{15}(5+3 n)(3+2 n)(2 n+1) !, \\
f(0, \pm, 0, \pm, n) & =\left\langle 0\left|(\theta)^{n} a_{ \pm} a_{0} a_{ \pm}^{\dagger} a_{0}^{\dagger}\left(\theta^{\dagger}\right)^{n}\right| 0\right\rangle \\
& =\frac{1}{15}(5+2 n)(3+2 n)(2 n+1) !, \\
f(+,-,+,-, n) & =\left\langle 0\left|(\theta)^{n} a_{+} a_{-} a_{+}^{\dagger} a_{-}^{\dagger}\left(\theta^{\dagger}\right)^{n}\right| 0\right\rangle \\
& =\frac{1}{15}(5+4 n)(3+2 n)(2 n+1) !, \\
f(+,-, 0,0, n) & =\left\langle 0\left|(\theta)^{n} a_{+} a_{-} a_{0}^{\dagger} a_{0}^{\dagger}\left(\theta^{\dagger}\right)^{n}\right| 0\right\rangle \\
& =-\frac{2}{15} n(3+2 n)(2 n+1) ! .
\end{aligned}
$$

\section{Calculation of $\epsilon_{0}$}

So, first let us calculate the energy for total spin 0 state. From known Clebsch-Gordon coefficients, this state is

$$
\begin{aligned}
\left|S=0, S_{z}=0\right\rangle= & \frac{1}{\sqrt{3}}\left(|1,1\rangle_{i}|1,-1\rangle_{j}-|1,0\rangle_{i}|1,0\rangle_{j}\right. \\
& \left.+|1,-1\rangle_{i}|1,1\rangle_{j}\right)
\end{aligned}
$$

Rewriting it via creation operators, we get the normalized state

$$
\left|S_{i}+S_{j}=0\right\rangle=\frac{1}{\sqrt{3} f(n, 1)} a_{i, p}^{\dagger} a_{j, p}^{\dagger}\left(\theta_{i}^{\dagger}\right)^{n}\left(\theta_{j}^{\dagger}\right)^{n}|0\rangle .
$$

In the second order of perturbation theory, the energy expectation value is

$$
\sum_{m}-\frac{t^{2}}{E_{m}-E_{0}}\left|\left\langle m\left|\left(a_{i p}^{\dagger} a_{j p}+a_{j p}^{\dagger} a_{i p}\right)\right| S_{i}+S_{j}\right\rangle\right|^{2} .
$$

The intermediate state $|m\rangle$ cannot correspond to $S_{i}=2, S_{j}$ $=0$ since in this case two spins cannot add to form a singlet. There are always at least two possible states:

$$
S_{i}=0, n_{i}=2 n+2, S_{j}=0, n_{j}=2 n, S_{i}+S_{j}=0
$$

and $i \leftrightarrow j$. The matrix element for each of these states is

$$
\begin{aligned}
& \frac{1}{\sqrt{3} f(n, 1)}\left\langle 0\left|\theta^{n+1} a_{p}^{\dagger} a_{q}^{\dagger}\left(\theta^{\dagger}\right)^{n}\right| 0\right\rangle_{i}\left\langle 0\left|\theta^{n} a_{p} a_{q}^{\dagger}\left(\theta^{\dagger}\right)^{n}\right| 0\right\rangle_{j} \\
& \times \frac{1}{\sqrt{[f(n+1,0) f(n, 0)]}} \\
& =\frac{1}{\sqrt{3} f(n, 1)}\left\langle 0\left|\theta^{n+1} a_{p}^{\dagger} a_{q}^{\dagger}\left(\theta^{\dagger}\right)^{n}\right| 0\right\rangle_{i} \delta_{p q} f(n, 1)
\end{aligned}
$$




$$
\begin{aligned}
& \times \frac{1}{\sqrt{[f(n+1,0) f(n, 0)]}} \\
= & \sqrt{\left(\frac{f(n+1,0)}{3 f(n, 0)}\right)} .
\end{aligned}
$$

Finally, this term gives

$$
-\frac{2 t^{2}}{3\left(U_{0}-2 U_{2}\right)} \frac{f(n+1,0)}{f(n, 0)}=-\frac{4 t^{2}(n+1)(2 n+3)}{3\left(U_{0}-2 U_{2}\right)} .
$$

In the general case $n \neq 0$ there can also be intermediate states

$$
S_{i}=2, n_{i}=2 n+2, S_{j}=2, n_{j}=2 n, S_{i}+S_{j}=0
$$

and $i \leftrightarrow j$ because $S_{i}=2$ and $S_{j}=2$ can form a singlet. However, in the case of $n=0$, such a term does not exist but can appear if $a_{j p}$, acting on $a_{j q}^{\dagger}\left(\theta_{j}^{\dagger}\right)^{n}|0\rangle$, breaks a singlet part and the result has a mixture of $S=2$. But for $n=0$ there is no singlet part, so these intermediate states are absent. One should also note that even for big $n$ we will not have terms with higher spins, i.e., $S_{i}=4$, because our perturbation in the Hilbert space of $i$ th site is a vector and can have matrix elements only for the states with spins differing by \pm 1 .

From general considerations we know that for $S_{i}+S_{j}=0$ energy has the form

$$
-2 t^{2}\left(\frac{f_{1}(n)}{U_{0}-2 U_{2}}+\frac{f_{2}(n)}{U_{0}+4 U_{2}}\right),
$$

where the term with $U_{0}-2 U_{2}$ in the denominator comes from the processes of the first kind, and the term with $U_{0}$ $+4 U_{2}$ in the denominator comes from the processes of the second kind. We calculated $f_{1}(n)$ earlier to be $2(n+1)(2 n$ $+3) / 3$. Now, to find $f_{2}(n)$, we take the limit $U_{2} \rightarrow 0$. In this case we do not need to know the exact form of states with $S_{i}=S_{j}=0$ and $S_{i}=S_{j}=2, S_{i}+S_{j}=0$ since their energy is the same. We can take the intermediate state $|m\rangle$ to be

$$
|m\rangle=\frac{1}{\sqrt{M}}\left(a_{i, p}^{\dagger} a_{j, p}+a_{j, p}^{\dagger} a_{i, p}\right) a_{i, q}^{\dagger} a_{j, q}^{\dagger}\left(\theta_{i}^{\dagger}\right)^{n}\left(\theta_{j}^{\dagger}\right)^{n}|0\rangle,
$$

where $M$ is normalization of the intermediate state. Then, the second-order energy is

$$
-t^{2} \frac{\left|\langle m \mid m\rangle \frac{\sqrt{M}}{\sqrt{3} f(n, 1)}\right|^{2}}{U_{0}}=-\frac{t^{2}}{U_{0}} \frac{M}{3 f(n, 1)^{2}} .
$$

Using formulas from the previous subsection, we can calculate $M$. Then, we can write an equation

$$
2\left[f_{1}(n)+f_{2}(n)\right]=\frac{M}{3 f(n, 1)^{2}}=2+\frac{6}{5} n(5+2 n)
$$

and finally obtain

$$
\epsilon_{0}=-\frac{4 t^{2}(n+1)(2 n+3)}{3\left(U_{0}-2 U_{2}\right)}-\frac{16 t^{2} n(5+2 n)}{15\left(U_{0}+4 U_{2}\right)} .
$$

\section{Calculation of $\epsilon_{1}$}

Let us consider the case $S_{i}+S_{j}=1$. There are no intermediate states $S_{i}=0, S_{j}=2$ or $S_{i}=0, S_{j}=0$ since these states can only form $S_{i}+S_{j}=2$ and $S_{i}+S_{j}=0$, respectively. $S_{i}=2, S_{j}$ $=2$ can add up to form $S_{j}+S_{j}=1$, and this is the only contribution. $S_{i}+S_{j}=1$ subspace is three dimensional, and from rotational invariance we can choose any state we want to calculate the energy. Let us choose our initial state to be $S_{i}$ $=1, S_{j}=1, S_{i}+S_{j}=1, S_{i z}+S_{j z}=0$. From known ClebschGordon coefficients, we can write this state using creation and annihilation operators as

$$
\left|S_{i}+S_{j}=1\right\rangle=\frac{a_{i,+}^{\dagger} a_{j,-}^{\dagger}-a_{i,-}^{\dagger} a_{j,+}^{\dagger}}{\sqrt{2} f(n, 1)}\left(\theta_{i}^{\dagger}\right)^{n}\left(\theta_{j}^{\dagger}\right)^{n}|0\rangle .
$$

Normalization of

$$
\begin{aligned}
& \left(a_{i,+}^{\dagger} a_{j,+}+a_{i, 0}^{\dagger} a_{j, 0}+a_{i,-}^{\dagger} a_{j,-}\right) \\
& \quad \times\left(a_{i,+}^{\dagger} a_{j,-}^{\dagger}-a_{i,-}^{\dagger} a_{j,+}^{\dagger}\right)\left|S_{i}+S_{j}=1\right\rangle
\end{aligned}
$$

equals

$$
\begin{aligned}
2[f( & +, 0, n)(-2 n)^{2} f(+, 0, n-1) \\
& \left.+f(+,+, n)(2 n)^{2} f(+,+, n-1)\right] \\
& =\frac{4}{45} n(1+2 n)(3+2 n)^{2}(5+2 n)(2 n+1) !^{2} .
\end{aligned}
$$

Hence, the energy for this case is

$$
\epsilon_{1}=-\frac{4 t^{2} n(5+2 n)}{5\left(U_{0}+4 U_{2}\right)}
$$

\section{Calculation of $\epsilon_{2}$}

Let us choose the normalized state for which we will calculate the second order energy to be the state with total $S_{i z}$ $+S_{j z}=2$ :

$$
\left|S_{i}+S_{j}=2\right\rangle=\frac{1}{f(n, 1)} a_{i,+}^{\dagger} a_{j,+}^{\dagger}\left(\theta_{i}^{\dagger}\right)^{n}\left(\theta_{j}^{\dagger}\right)^{n}|0\rangle .
$$

The intermediate state should also have $S_{i}+S_{j}=2, S_{i z}+S_{j z}$ $=2$. Such a state cannot belong to $S_{i}=0, S_{j}=0$ subspace since this pair of spins cannot add up to form $S_{i}+S_{j}=2$. There are four possible intermediate states with

$$
\begin{gathered}
S_{i}=2, n_{i}=2 n+2, S_{j}=0, n_{j}=2 n, \\
S_{i}=0, n_{i}=2 n+2, S_{j}=2, n_{j}=2 n,
\end{gathered}
$$

and $i \leftrightarrow j$. In the first case, matrix element equals 


$$
\begin{aligned}
& \frac{1}{f(n, 1)}\left\langle 0\left|\theta^{n} a_{+} a_{+} a_{+}^{\dagger} a_{+}^{\dagger}\left(\theta^{\dagger}\right)^{n}\right| 0\right\rangle_{i}\left\langle 0\left|\theta^{n} a_{+} a_{+}^{\dagger}\left(\theta^{\dagger}\right)^{n}\right| 0\right\rangle_{j} \\
& \quad \times \frac{1}{\sqrt{f(n, 2) f(n, 0)}} \\
& =\frac{1}{f(n, 1)} f(n, 2) f(n, 1) \frac{1}{\sqrt{[f(n, 2) f(n, 0)]}} \\
& =\sqrt{\left(\frac{f(n, 2)}{f(n, 0)}\right)} .
\end{aligned}
$$

Finally, this term contributes to the energy

$$
-\frac{2 t^{2}}{\left(U_{0}+U_{2}\right)} \frac{f(n, 2)}{f(n, 0)}=-\frac{4 t^{2}(2 n+3)(2 n+5)}{15\left(U_{0}+U_{2}\right)} .
$$

The second process contributes with the same energy denominator but with a different dependence on $n$ :

$$
-\frac{16 t^{2} n(n+1)}{15\left(U_{0}+U_{2}\right)}
$$

Now, let us consider the contribution from the $S_{i}=2, S_{j}$ $=2, S_{i}+S_{j}=2$ intermediate state. Using the same trick as at the end of the calculation of $\epsilon_{0}$, we only need to calculate norm of

$$
\left(a_{i,+}^{\dagger} a_{j,+}+a_{i, 0}^{\dagger} a_{j, 0}+a_{i,-}^{\dagger} a_{j,-}\right) a_{i,+}^{\dagger} a_{j,+}^{\dagger}\left(\theta_{i}^{\dagger}\right)^{n}\left(\theta_{j}^{\dagger}\right)^{n}|0\rangle .
$$

This quantity equals

$$
\begin{aligned}
f(+,+, n)[f(0,0, n-1)-2(2 n+2) f(+,-, 0, n-1) \\
\left.+(2 n+2)^{2} f(+,-, n-1)\right]+f(+, 0, n)(2 n) \\
\quad \times^{2} f(+,-, n-1)+f(+,-, n)(-2 n)^{2} f(+,+, n-1) \\
=\frac{2}{225}(3+2 n)^{2}(5+3 n)(5+6 n)(2 n+1) !^{2} .
\end{aligned}
$$

Then, taking a limit $U_{2} \rightarrow 0$, we obtain the contribution from this process to be

$$
-\frac{28 t^{2} n(5+2 n)}{75\left(U_{0}+4 U_{2}\right)}
$$

Finally,

$$
\epsilon_{2}=-\frac{28 t^{2} n(5+2 n)}{75\left(U_{0}+4 U_{2}\right)}-\frac{4\left(15+20 n+8 n^{2}\right)}{15\left(U_{0}+U_{2}\right)} .
$$

\section{APPENDIX B: DERIVATION OF THE EFFECTIVE MAGNETIC HAMILTONIAN FOR THE INSULATING STATE WITH TWO ATOMS}

To derive the effective Hamiltonian, we should be able to calculate matrix elements in Eq. (31). Since energy and matrix elements in each subspace do not depend on $z$ projection of total spin, we can choose $S_{z}$ components at our conve- nience. We can express any state $\left|E_{1}\right\rangle, \ldots,\left|E_{8}\right\rangle$ using known Clebsch-Gordon coefficients. For the state from $\left|E_{8}\right\rangle$ with $S_{z}=0$, we have

$$
\begin{aligned}
\left|E_{8}, S_{z}\right\rangle= & \sum_{m=-2, \ldots, 2} C_{2, m, 2,-m}^{4,0}\left|N_{1}=2, S_{1}=2, S_{1 z}=m\right\rangle \\
& \times\left|N_{2}=2, S_{2}=2, S_{2 z}=-m\right\rangle .
\end{aligned}
$$

Now we can write any one of the states $\left|N_{i}, S_{i}, S_{i z}\right\rangle$ via creation and annihilation operators since we know how to express spin operators via creation and annihilation operators (11). Evaluation of $\tilde{e}_{6}-\tilde{e}_{8}$ is quite simple since total spin conservation of the tunneling term does not allow mixing of this subspaces with any other. Therefore, as in Eq. (A2), we just need to calculate the normalization of the state into which we hop. Using this procedure, we obtain energies (30).

Now let us consider energy in the $\left|E_{1}\right\rangle,\left|E_{2}\right\rangle$ subspace. From $\left|E_{1}\right\rangle$ we can hop only into high-energy states

$$
\begin{aligned}
& \left|N_{1}=3, N_{2}=1, S_{1}=S_{2}=1, S_{1}+S_{2}=0\right\rangle, \\
& \left|N_{1}=1, N_{2}=3, S_{1}=S_{2}=1, S_{1}+S_{2}=0\right\rangle,
\end{aligned}
$$

since in the Hilbert space of each well spin can change only by \pm 1 . For $N=2$ from $\left|E_{2}\right\rangle$, we can also tunnel only to these states since 3 and 1 cannot add to form total spin 0 , and there is no state $S_{1}=S_{2}=3$ which can also add up to total spin 0 . Therefore, our exact Hamiltonian in the basis of $\left|E_{1}\right\rangle,\left|E_{2}\right\rangle$ and high-energy states (B1) has the form

$$
\left[\begin{array}{cccc}
0 & 0 & V_{1} & V_{1} \\
0 & 6 U_{2} & V_{2} & V_{2} \\
V_{1} & V_{2} & U_{0} & 0 \\
V_{1} & V_{2} & 0 & U_{0}
\end{array}\right] .
$$

We can diagonalize this Hamiltonian in the low-energy $\left|E_{1}\right\rangle,\left|E_{2}\right\rangle$ subspace in the limit

$$
V_{1}, V_{2} \ll U_{0}, U_{2} \ll U_{0} .
$$

First, we integrate out high energy levels - this is done as described in Ref. [1]. We use the following matrix identity:

$$
\left[\left(\begin{array}{ll}
A & B \\
C & D
\end{array}\right)^{-1}\right]_{i j}=\left[\left(A-B D^{-1} C\right)^{-1}\right]_{i j},
$$

where $1 \leqslant i, j \leqslant 2$.

In our case, $D$ has the form $U_{0} I_{2}$, so it is easy to calculate inverse matrix. Finally, our effective Hamiltonian has the form

$$
\left[\begin{array}{cc}
0 & 0 \\
0 & 6 U_{2}
\end{array}\right]-\frac{2}{U_{0}}\left[\begin{array}{cc}
V_{1}^{2} & V_{1} V_{2} \\
V_{1} V_{2} & V_{2}^{2}
\end{array}\right] .
$$

Now we can diagonalize this $2 \times 2$ matrix; its energy levels are

$$
3 U_{2}-\frac{V_{1}^{2}+V_{2}^{2}}{U_{0}} \pm \sqrt{\left(3 U_{2}-\frac{V_{1}^{2}+V_{2}^{2}}{U_{0}}\right)^{2}+12 \frac{U_{2} V_{1}^{2}}{U_{0}}}
$$


Using expressions for all states of interest in Fock basis, we can calculate

$$
V_{1}=-t \sqrt{\frac{10}{3}}, \quad V_{2}=-t \sqrt{\frac{8}{3}},
$$

which leads to Eqs. (20), (21).

Now, let us calculate energy for the $S_{i}+S_{j}=2$ subspace. In this case we can hop to four states

$$
\begin{gathered}
\left|N_{1}=3, N_{2}=1, S_{1}=S_{2}=1, S_{1}+S_{2}=2\right\rangle, \\
\left|N_{1}=1, N_{2}=3, S_{1}=S_{2}=1, S_{1}+S_{2}=2\right\rangle, \\
\left|N_{1}=1, N_{2}=3, S_{1}=1, S_{2}=3, S_{1}+S_{2}=2\right\rangle, \\
\left|N_{1}=3, N_{2}=1, S_{1}=3, S_{2}=1, S_{1}+S_{2}=2\right\rangle .
\end{gathered}
$$

$$
\left[\begin{array}{ccc}
3 U_{2} & 0 & 0 \\
0 & 3 U_{2} & 0 \\
0 & 0 & 6 U_{2}
\end{array}\right]-\frac{1}{U_{0}}\left[\begin{array}{c}
V_{1}^{2}+V_{2}^{2}+V_{3}^{2} \\
2 V_{1} V_{2} \\
\left(V_{1}+V_{2}\right) V_{4}+V_{3} V_{5}
\end{array}\right.
$$

$$
\left.\begin{array}{cc}
2 V_{1} V_{2} & \left(V_{1}+V_{2}\right) V_{4}+V_{3} V_{5} \\
V_{1}^{2}+V_{2}^{2}+V_{3}^{2} & \left(V_{1}+V_{2}\right) V_{4}+V_{3} V_{5} \\
\left(V_{1}+V_{2}\right) V_{4}+V_{3} V_{5} & 2\left(V_{4}^{2}+V_{5}^{2}\right)
\end{array}\right]
$$

and $C=B^{T}$. The effective Hamiltonian is . and matrix $\mathrm{B}$ has the form

$$
\left[\begin{array}{cccc}
V_{1} & V_{2} & V_{3} & 0 \\
V_{2} & V_{1} & 0 & V_{3} \\
V_{4} & V_{4} & V_{5} & V_{5}
\end{array}\right]
$$


The numerical procedure now consists of fixing the values of $t$ and $\theta$ and minimizing the expression for energy over six angles using steepest descents method. Using this procedure we can numerically find energy as a function of $\theta$ for fixed $t$. If the minimum is attained at $\theta=0$, then the mean-field wave function is a trivial singlet. Result of this minimization leads to the state

$$
b_{m}=\left(\frac{1}{\sqrt{6}}, \pm \frac{1}{\sqrt{3}}, 0, \mp \frac{1}{\sqrt{3}}, \frac{1}{\sqrt{6}}\right)^{T}
$$

that can be rewritten as Eq. (42) after rotation. Though this result was obtained numerically, we can check analytically that at this point all first derivatives of the energy over angles $\psi, \psi_{1}$, and $\psi_{2}$ vanish.

\section{APPENDIX D: LARGE $N$ EXPANSION}

In this Appendix we prove some properties of wave functions $|\psi\rangle_{N}$ (59). The normalization factor $\mathcal{N}$ in Eq. (59) may be found by considering the overlap of two states. It is sufficient to consider wave functions constructed of only two single particle states since rotation in the spinor state can always bring our "pure condensate" wave functions to this case:

$$
\begin{aligned}
& \left|N, \mathbf{n}_{1}\right\rangle=\frac{1}{\mathcal{N}}\left(\cos \phi_{1} a_{x}^{\dagger}+\sin \phi_{1} a_{y}^{\dagger}\right)^{N}|0\rangle \\
& \left|N, \mathbf{n}_{2}\right\rangle=\frac{1}{\mathcal{N}}\left(\cos \phi_{2} a_{x}^{\dagger}+\sin \phi_{2} a_{y}^{\dagger}\right)^{N}|0\rangle .
\end{aligned}
$$

We have

$$
\begin{aligned}
\left\langle N, \mathbf{n}_{1} \mid N, \mathbf{n}_{2}\right\rangle= & \frac{1}{\mathcal{N}^{2}}\langle 0|\left(\cos \phi_{1} a_{x}+\sin \phi_{1} a_{y}\right)^{N}\left(\cos \phi_{2} a_{x}^{\dagger}\right. \\
& \left.+\sin \phi_{2} a_{y}^{\dagger}\right)^{N}|0\rangle \\
= & \frac{1}{\mathcal{N}^{2}} \sum_{k=0}^{N}\left(C_{N}^{k}\right)^{2}\left(\cos \phi_{1} \cos \phi_{2}\right)^{k}
\end{aligned}
$$

$$
\begin{aligned}
& \times\left(\sin \phi_{1} \sin \phi_{2}\right)^{N-k} \\
& \times\left\langle 0\left|a_{x}^{k} a_{y}^{N-k}\left(a_{x}^{\dagger}\right)^{k}\left(a_{y}^{\dagger}\right)^{N-k}\right| 0\right\rangle \\
= & \frac{N !}{\mathcal{N}^{2}} \sum_{k=0}^{N} C_{N}^{k}\left(\cos \phi_{1} \cos \phi_{2}\right)^{k}\left(\sin \phi_{1} \sin \phi_{2}\right)^{N-k} \\
= & \frac{N !}{\mathcal{N}^{2}} \cos ^{N}\left(\phi_{1}-\phi_{2}\right) \\
= & \frac{N !}{\mathcal{N}^{2}}\left(\mathbf{n}_{1} \cdot \mathbf{n}_{2}\right)^{N} .
\end{aligned}
$$

Orthogonality and normalization for large $N$ now become obvious after noting that for $\left(\mathbf{n}_{1} \cdot \mathbf{n}_{2}\right)=\cos \theta$ and $\theta \leqslant \pi / 2$ we have $\cos ^{N} \theta \approx e^{-N \theta^{2} / 2}$.

To prove Eq. (65) we consider wave functions

$$
\left|N+1, \mathbf{n}^{\prime}\right\rangle=\frac{1}{\mathcal{N}_{N+1}}\left(n_{x}^{\prime} a_{x}^{\dagger}+n_{y}^{\prime} a_{y}^{\dagger}\right)^{N}|0\rangle .
$$

Simple calculation gives

$$
\begin{aligned}
\left\langle N+1, \mathbf{n}^{\prime}\left|a_{x}^{\dagger}\right| N, \mathbf{n}\right\rangle & =\frac{(N+1) !}{\mathcal{N}_{N} \mathcal{N}_{N+1}} n_{x}^{\prime}\left(\mathbf{n} \cdot \mathbf{n}^{\prime}\right)^{N} \\
& =(N+1)^{1 / 2} n_{x} \delta_{N}\left(\mathbf{n}-\mathbf{n}^{\prime}\right) .
\end{aligned}
$$

[1] A. Auerbach, Interacting Electrons and Quantum Magnetism (Springer-Verlag, Berlin, 1994).

[2] I. Affleck, Nucl. Phys. B265, 409 (1984).

[3] C. Nayak and K. Shtengel, Phys. Rev. B 64, 064422 (2001).

[4] S.A. Kivelson, Synth. Met. 125, 99 (2002).

[5] D.A. Ivanov and T. Senthil, Phys. Rev. B 66, 115111 (2002).

[6] M. Levin and X.-G. Wen, cond-mat/0302460 (unpublished).

[7] M. Hermele, M.P.A. Fisher, and L. Balents, e-print cond-mat/0305401 (unpublished).

[8] A.A. Nersesyan and A.M. Tsvelik, e-print cond-mat/0206483 (unpublished).

[9] R. Moessner, S.L. Sondhi, and E. Fradkin, Phys. Rev. B 65, 024504 (2002).

[10] L. Balents, M.P.A. Fisher, and S.M. Girvin, e-print cond-mat/0110005 (unpublished).
[11] C.J. Pethick and H. Smith, Bose-Einstein Condensation in Dilute Gases (Cambridge University Press, Cambridge, UK, 2002).

[12] K. Burnett et al., Nature (London) 416, 225 (2002).

[13] C. Orzel et al., Science 291, 2386 (2001).

[14] M. Greiner et al., Nature (London) 415, 39 (2002).

[15] S. Rolston and W. Phillips (private communication).

[16] D. Jaksch et al., Phys. Rev. Lett. 81, 3108 (1998).

[17] M.P.A. Fisher et al., Phys. Rev. B 40, 546 (1989).

[18] T.L. Ho, Phys. Rev. Lett. 81, 742 (1998).

[19] T. Ohmi and K. Machida, J. Phys. Soc. Jpn. 67, 1822 (1998).

[20] Y. Castin and C. Herzog, e-print cond-mat/0012040 (unpublished).

[21] T.L. Ho and S.K. Yip, Phys. Rev. Lett. 84, 4031 (2000).

[22] C.K. Law, H. Pu, and N.P. Bigelow, Phys. Rev. Lett. 81, 5257 
(1998).

[23] W.V. Liu and X.G. Wen, e-print cond-mat/0201187 (unpublished)

[24] D.M. Stamper-Kurn et al., Phys. Rev. Lett. 80, 2027 (1998).

[25] A.B. Kuklov and B.V. Svistunov, Phys. Rev. Lett. 90, 100401 (2003).

[26] L.-M. Duan, E. Demler, and M.D. Lukin, e-print cond-mat/0210564 (unpublished).

[27] E. Demler and F. Zhou, Phys. Rev. Lett. 88, 163001 (2002).

[28] F. Zhou, e-print cond-mat/0108473 (unpublished); e-print cond-mat/0207041 (unpublished).

[29] S.K. Yip, e-print physics/0306018 (unpublished).

[30] E. Demler et al., Phys. Rev. B 65, 155103 (2002).

[31] J.P. Burke, Jr., C.H. Greene, and J.L. Bohn, Phys. Rev. Lett. 81, 3355 (1998)

[32] S. Coleman, Commun. Math. Phys. 31, 259 (1973).

[33] A.V. Chubukov, J. Phys.: Condens. Matter 2, 1593 (1990); A.V. Chubukov, Phys. Rev. B 43, 3337 (1991).

[34] B.A. Ivanov and A.K. Kolezhuk, e-print cond-mat/0205207 (unpublished).

[35] G. Fath and J. Solyom, Phys. Rev. B 51, 3620 (1995).

[36] H.H. Chen and P.M. Levy, Phys. Rev. B 7, 4267 (1973).

[37] N. Papanicoulaou, Nucl. Phys. B305, 367 (1988).

[38] K. Harada and N. Kawashima, e-print cond-mat/0011346 (unpublished); Phys. Rev. B 65, 052403 (2002).
[39] K. Tanaka, A. Tanaka, and T. Idogaki, J. Phys. A 34, 8767 (2001).

[40] P.G. de Gennes and J. Prost, The Physics of Liquid Crystals (Oxford Press, Oxford, 1993).

[41] A.F. Andreev and I.A. Grischuk, Zh. Eksp. Teor. Fiz. 87, 467 (1984).

[42] Expression (42) describes an eigenstate of $S^{z}$ with eigenvalue zero. After an $\mathrm{SU}(2)$ rotation we will have $\left[a_{m}\right]$ that is an eigenstate of $\vec{d} \cdot \vec{S}$ with zero eigenvalue. Vector $\vec{d}$ corresponds to the direction of uniaxial nematic.

[43] M. Greiner et al., e-print cond-mat/0207196 (unpublished).

[44] E. Demler, F. Zhou, and F.D.M. Haldane, Report No. ITP-UU01/09, 2001.

[45] The operator $b$ applied directly to $|\psi\rangle_{N}$ takes us outside of the physical Hilbert space since it does not change the symmetry of the $\psi(\mathbf{n})$ wave function simultaneously with changing the number of particles by one. The physical Hamiltonian, however, will always have this operator $b$ in combination with some odd function of $\mathbf{n}$ and will preserve the physical Hilbert space.

[46] S. Tsuchiya, S. Kurihara, and T. Kimura, e-print cond-mat/0209676 (unpublished).

[47] D.M. Stamper-Kurn et al., Phys. Rev. Lett. 83, 2876 (1999).

[48] M. Snoek and F. Zhou, e-print cond-mat/0306198 (unpublished).

[49] J.W. Negele and H. Orland, Quantum Many-Particle Systems (Addison-Wesley, New York, 1988). 